\title{
A new formulation for air-blast fluid-structure interaction using an immersed approach: part II-coupling of IGA and meshfree discretizations
}

\author{
Y. Bazilevs ${ }^{1}$ - G. Moutsanidis ${ }^{1}$ - J. Bueno ${ }^{3}$ - K. Kamran ${ }^{1}$ - D. Kamensky ${ }^{1}$ \\ M. C. Hillman ${ }^{2}$ - H. Gomez ${ }^{4}$ - J. S. Chen ${ }^{1}$
}

Received: 3 December 2016 / Accepted: 20 February 2017 / Published online: 14 March 2017

(C) Springer-Verlag Berlin Heidelberg 2017

\begin{abstract}
In this two-part paper we begin the development of a new class of methods for modeling fluid-structure interaction (FSI) phenomena for air blast. We aim to develop accurate, robust, and practical computational methodology, which is capable of modeling the dynamics of air blast coupled with the structure response, where the latter involves large, inelastic deformations and disintegration into fragments. An immersed approach is adopted, which leads to an a-priori monolithic FSI formulation with intrinsic contact detection between solid objects, and without formal restrictions on the solid motions. In Part I of this paper, the core air-blast FSI methodology suitable for a variety of discretizations is presented and tested using standard finite elements. Part II of this paper focuses on a particular instantiation of the proposed framework, which couples isogeometric analysis (IGA) based on non-uniform rational B-splines and a reproducing-kernel particle method (RKPM), which is a meshfree technique. The combination of IGA and RKPM is felt to be particularly attractive for the problem class of interest due to the higher-order accuracy and smoothness of both discretizations, and relative simplicity of RKPM in handling fragmentation scenarios. A collection of mostly $2 \mathrm{D}$ numeri-
\end{abstract}

$\triangle$ Y. Bazilevs

yuri@ucsd.edu

1 Department of Structural Engineering, University of California, San Diego, La Jolla, CA 92093, USA

2 Department of Civil and Environmental Engineering, Pennsylvania State University, State College, PA 16801, USA

3 Departamento de Metodos Matematicos e de Representacion, Universidade da Coruña, Campus de Elviña, 15071 A Coruña, Spain

4 School of Mechanical Engineering, Purdue University, 585 Purdue Mall, West Lafayette, IN 47907, USA cal examples is presented in each of the parts to illustrate the good performance of the proposed air-blast FSI framework.

Keywords Air blast · FSI · Immersed methods · FEM . IGA $\cdot$ RKPM

\section{Introduction}

In Part I of this paper we proposed a computational fluidstructure interaction (FSI) framework, which is based on the immersed methodology, and which is aimed at modeling the dynamics of air blast coupled with the structure response involving large, inelastic deformations, and disintegration into fragments. In the proposed framework, the Navier-Stokes equations of compressible flows are coupled with those of a large-deformation inelastic solid. Two discretizations, background and foreground, are employed in the formulation: A fixed background discretization provides the basis functions used to approximate the unknowns of the coupled FSI problem; A foreground discretization moving with the solid material particles tracks the solid current position, stores the history-dependent variables, and is employed to perform numerical quadrature for the solid terms. The formulation was successfully tested using the standard loworder finite-element discretization.

Here, in Part II of the manuscript we focus on a particular instantiation of the proposed framework, which couples isogeometric analysis (IGA) [1,2] based on non-uniform rational B-splines (NURBS) [3-5] as the background discretization, and the reproducing-kernel particle method (RKPM) [6-9] as the foreground discretization. This combination is particularly attractive for the problem class of interest due to the higher-order accuracy and higher degree of continuity inherent in both techniques, and the relative 
simplicity of RKPM in handling the solid large-deformation and fragmentation scenarios. Higher-order accuracy and smoothness of the discretizations was shown to be beneficial for both fluid and solid/structural mechanics applications, both in terms of accuracy $[10,11]$ and robustness [12] of the resulting discrete solutions. In addition, in the present framework, higher-order smoothness of the background discretization yields a continuous representation of the strain rate, and thus precludes jumps in the stress and other history variables as the Lagrangian particles cross the backgroundelement boundaries. We note that the strain-rate discontinuity across element boundaries is recognized as a shortcoming of FEM-based material-point-type methods [13], which may be naturally overcome using IGA for the background discretization.

The paper is outlined as follows. In Sect. 2 we review the basics of NURBS-based IGA. In Sect. 3 we give a brief overview of Meshfree methods, with an emphasis on RKPM and domain-integration techniques. In Sect. 4 we present the items speeific to the IGA-RKPM coupling for air-blast FSI. In Sect. 5 we compute several numerical examples that demonstrate the good accuracy, stability, and robustness of the proposed formulation. While a simulation showing mul-

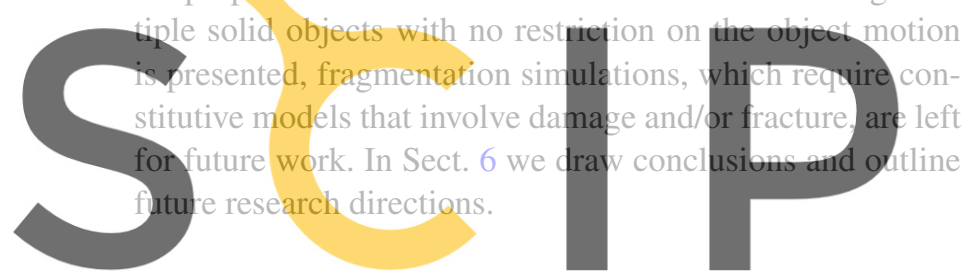

2 NURBS-based IGA

Register for free at https//www.scipedia.com to

In this section we briefly recall the basics of IGA $[1,2]$ based on NURBS [3-5]. NURBS are convenient for free-form surface modeling, and can represent exactly all conic sections. In addition, they exhibit excellent mathematical properties, such as derivative-continuity across element boundaries, optimal approximation [14], and the ability to be refined through knot insertion and degree elevation. We note that in the present effort NURBS-based IGA is employed to provide a smooth background-mesh discretization, which has significant benefits compared to $C^{0}$-continuous approximations in many applications [11,14-18]. In the present work we do not directly take advantage of the geometric flexibility of IGA, although one may envision cases where background discretization conforming to geometrically-complex fluidmechanics domains may be desirable.

\subsection{B-splines}

NURBS are built from B-splines. A necessary component for the construction of B-splines is the knot vector. A knot vector in $1 \mathrm{D}$ is a non-decreasing set of coordinates in the parametric domain written as $\Xi=\left\{\xi_{1}, \xi_{2}, \ldots, \xi_{n+p+1}\right\}$, where $\xi_{i} \in \mathbb{R}$ is the $i$ th knot, $i$ is the knot index, $i=1,2, \ldots, n+p+1, p$ is the polynomial order, and $n$ is the number of $\mathrm{B}$-spline basis functions. Knots divide the parametric domain into elements.

For a given knot vector, the B-spline basis functions are defined recursively starting with piecewise constants $(p=0)$ :

$N_{i, 0}(\xi)= \begin{cases}1 & \text { if } \xi_{i} \leqslant \xi<\xi_{i+1} \\ 0 & \text { otherwise }\end{cases}$

For $p=1,2,3, \ldots$, they are defined by

$$
\begin{aligned}
N_{i, p}(\xi)= & \frac{\xi-\xi_{i}}{\xi_{i+p}-\xi_{i}} N_{i, p-1}(\xi) \\
& +\frac{\xi_{i+p+1}-\xi}{\xi_{i+p+1}-\xi_{i+1}} N_{i+1, p-1}(\xi),
\end{aligned}
$$

\section{which is the Cox-de Boor recursion formula $[19,20]$.}

Knot vectors may be open or closed. In an open knot vector the first and last knot values appear $p+1$ times. B-spline basis functions constructed using an open knot vector are interpolatory at the endpoints of the parametric interval, which facilitates imposition of boundary conditions. In general, B-

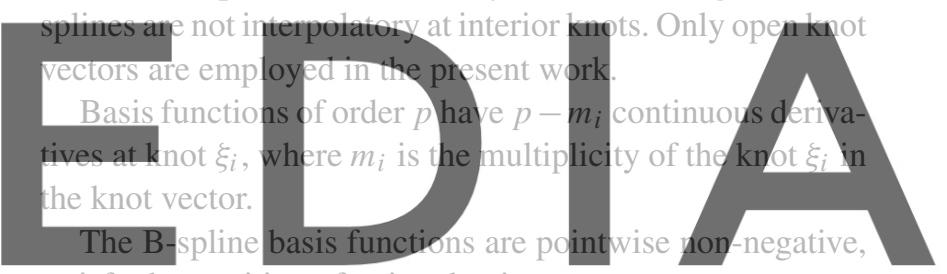

satisfy the partition of unity, that is,

\section{download the version without the watermark $\sum_{i=1} N_{i, p}(\xi)=1 \quad \forall \xi \in \Xi$,

and the support of each basis function $N_{i, p}$ is compact and contained in the interval $\left[\xi_{i}, \xi_{i+p+1}\right]$.

\subsection{NURBS}

Geometric entities in $\mathbb{R}^{\mathrm{d}}$ are obtained by a projective transformation of B-spline curves in $\mathbb{R}^{\mathrm{d}+1}$, where $\mathrm{d}$ is the space dimension. Conic sections may be exactly constructed by projective transformations of piecewise quadratic curves. This projective transformation gives rise to NURBS basis functions with the following structure:

$R_{i}^{p}(\xi)=\frac{N_{i, p}(\xi) w_{i}}{W(\xi)}$

where $W(\xi)$ is the weighting function given by

$W(\xi)=\sum_{i=1}^{n} N_{i, p}(\xi) w_{i}$, 
where $w_{i}$ 's are positive weights. As is evident from the above expressions, unlike B-spline or standard FEM basis functions that are piecewise polynomials, NURBS are piecewise rational functions.

NURBS curves are constructed using a linear combination of NURBS basis functions as

$\mathbf{C}(\xi)=\sum_{i=1}^{n} R_{i}^{p}(\xi) \mathbf{B}_{i}$,

where $\mathbf{B}_{i} \in \mathbb{R}^{\mathrm{d}}$ are the control points. Given additional knot vectors $H=\left\{\eta_{1}, \eta_{2}, \ldots, \eta_{m+q+1}\right\}$ and $Z=$ $\left\{\zeta_{1}, \zeta_{2}, \ldots, \zeta_{l+r+1}\right\}$, NURBS basis functions in 2D and 3D may be defined as

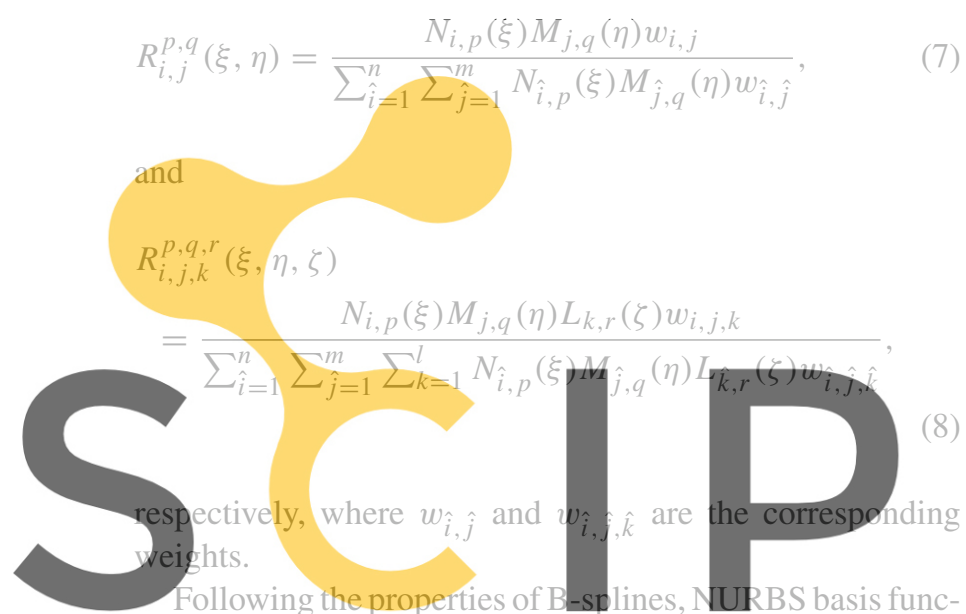

tions in all dimensions are pointwise nonnegative, form a

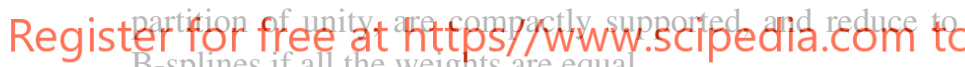
B-splines if all the weights are equal.

Analogously to NURBS curves, NURBS surfaces and volumes are defined as

$\mathbf{S}(\xi, \eta)=\sum_{i=1}^{n} \sum_{j=1}^{m} R_{i, j}^{p, q}(\xi, \eta) \mathbf{B}_{i, j}$

and

$\mathbf{V}(\xi, \eta, \zeta)=\sum_{i=1}^{n} \sum_{j=1}^{m} \sum_{k=1}^{l} R_{i, j, k}^{p, q, r}(\xi, \eta, \zeta) \mathbf{B}_{i, j, k}$,

respectively, where $\mathbf{B}_{i, j}$ 's and $\mathbf{B}_{i, j, k}$ form a control mesh.

\subsection{Analysis framework}

We write $\hat{N}(\boldsymbol{\xi})$ and $N(\mathbf{x})$ to refer to a generic NURBS basis function defined on the parametric and physical domains, respectively. We also make use of a single-index notation, and let indices $A, B, C, \ldots$ label the NURBS basis functions. In this setting, the geometry mapping may be expressed as
$\mathbf{x}(\boldsymbol{\xi})=\sum_{A=1}^{\mathrm{n}_{\mathrm{np}}} \mathbf{x}_{A} \hat{N}_{A}(\boldsymbol{\xi})$

where $n_{n p}$ denotes the number of control points in the mesh with coordinates given by $\mathbf{x}_{A}$ 's. This mapping may be restricted to a NURBS patch or element.

The IGA solution in the parametric domain, taken to be scalar-valued for the purposes of illustration, is assumed to be governed by the same NURBS basis functions, and may be expressed as

$\hat{u}^{h}(\boldsymbol{\xi})=\sum_{A=1}^{\mathrm{n}_{\mathrm{np}}} u_{A} \hat{N}_{A}(\boldsymbol{\xi})$,

where $u_{A}$ 's are the control variables or degrees of freedom (DOF). The IGA solution in the physical domain is defined as a push-forward of its parametric counterpart given by Eq. (12) by the geometrical mapping given by Eq. (11), and may be expressed as

\section{$u^{h}(\mathbf{x})=\sum_{A=1}^{\mathrm{n}_{\mathrm{np}}} u_{A} N_{A}(\mathbf{x})$}

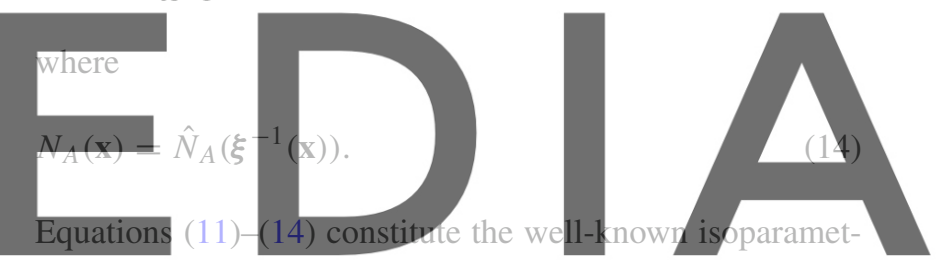

ric construction widely used in FEM and IGA. The above construction guarantees optimal approximation properties of dPWR Pad the versi:p without the watermark

The first and second partial derivatives of the basis functions in Eq. (14) with respect to physical coordinates, which are employed in the weak formulation of the FSI problem presented in Part I of this paper, are computed using the chain rule in a manner similar to FEM. To carry out the backgrounddomain weak-form integrals, standard Gaussian quadrature is employed. It should be noted that more efficient quadrature rules for IGA have been recently proposed (see, e.g., [22, 23]) and may be employed for better efficiency of the computational procedures. Dirichlet boundary conditions in IGA may be imposed strongly by selecting appropriate values of control variables belonging to essential boundaries, or weakly by means of Nitsche-like methods [24]. Because of the variational structure of IGA, Neumann boundary conditions are enforced weakly as in standard FEM.

\section{RKPM-based Meshfree methods}

Like IGA, Meshfree methods is a class of numerical methods that solve partial differential equations. They retain the useful 
characteristics of the FEM, such as good approximation properties and compact support of shape functions, but attempt to overcome some of the disadvantages, like mesh entanglement, material distortion, mesh dependency, and difficulty in constructing approximations with arbitrary order of continuity [6]. Meshfree methods share a common feature that no standard mesh data structures are required to define the discrete approximation spaces, which are constructed based on scattered points (or particles). These methods provide considerable advantages over traditional FEM for solving problems involving large deformations, damage, $h$-adaptive refinement, and evolving discontinuities. The reproducingkernel particle method (RKPM) [7-9,25] is a prominent representative of a class of Meshfree methods, and is presented in what follows.

\subsection{RKPM}

We assume that the problem domain (in our case, the solid domain $\Omega^{s}$ ) is discretized by a set of $\mathrm{n}_{\mathrm{np}}$ Lagrangian particles. An RKPM basis function associated with a given particle $A, \Psi_{A}(\mathbf{x})$, is constructed by multiplying a kernel function $\Phi_{a}\left(\mathbf{x}-\mathbf{x}_{A}\right)$ with a correction function $C\left(\mathbf{x}, \mathbf{x}-\mathbf{x}_{A}\right)$ as

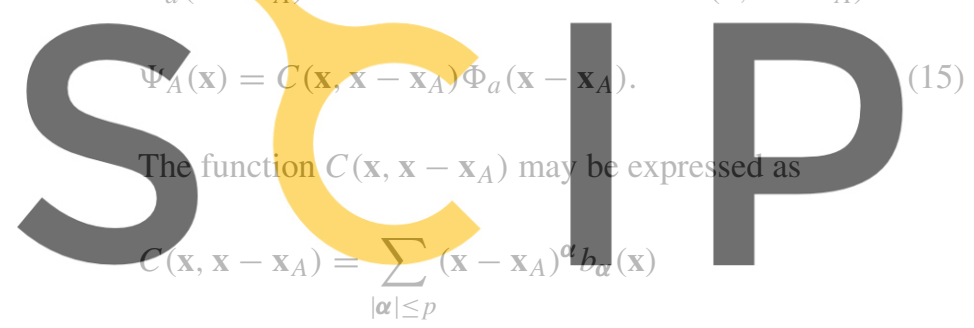

Register for free-att battps/fwwww.scipedia.coma to

where $\mathrm{p}\left(\mathrm{x}-\mathrm{x}_{A}\right)$ is the $p$ th-order monomial basis vector,

$\mathbf{b}(\mathbf{x})$ is the unknown coefficient vector, $\boldsymbol{\alpha}$ is a multi-index, and $|\boldsymbol{\alpha}|$ is its norm. The unknown coefficient vector $\mathbf{b}(\mathbf{x})$ is determined by imposing $p$ th-order polynomial reproducing conditions on the space spanned by RKPM basis functions, namely,

$$
\sum_{A=1}^{\mathrm{n}_{\mathrm{np}}} \Psi_{A}(\mathbf{x}) \mathbf{x}_{A}^{\boldsymbol{\alpha}}=\mathbf{x}^{\boldsymbol{\alpha}}, \quad|\boldsymbol{\alpha}| \leq p,
$$

or, equivalently,

$\sum_{A=1}^{\mathrm{n}_{\mathrm{np}}} \Psi_{A}(\mathbf{x}) \mathbf{p}\left(\mathbf{x}-\mathbf{x}_{A}\right)=\mathbf{p}(\mathbf{0})$.

Substituting the expression for $\Psi_{A}(\mathbf{x})$ from Eq. (15) into Eq. (18), and solving for $\mathbf{b}(\mathbf{x})$, gives

$$
\mathbf{b}(\mathbf{x})=\mathbf{M}^{-1}(\mathbf{x}) \mathbf{p}(\mathbf{0}),
$$

where the moment matrix $\mathbf{M}(\mathbf{x})$ is given by

$\mathbf{M}(\mathbf{x})=\sum_{A=1}^{\mathrm{n}_{\mathrm{np}}} \mathbf{p}\left(\mathbf{x}-\mathbf{x}_{A}\right) \mathbf{p}^{T}\left(\mathbf{x}-\mathbf{x}_{A}\right) \Phi_{a}\left(\mathbf{x}-\mathbf{x}_{A}\right)$.

The moment matrix is invertible at location $\mathbf{x}$ if the reproducing conditions given by Eq. (17) are linearly independent [25, 26]. An explicit form of the RKPM basis functions is obtained by substituting the result from Eq. (19) into Eq. (15):

$\Psi_{A}(\mathbf{x})=\mathbf{p}^{T}(\mathbf{0}) \mathbf{M}^{-1}(\mathbf{x}) \mathbf{p}\left(\mathbf{x}-\mathbf{x}_{A}\right) \Phi_{a}\left(\mathbf{x}-\mathbf{x}_{A}\right)$.

For the kernel function we choose a radial cubic B-spline, namely,

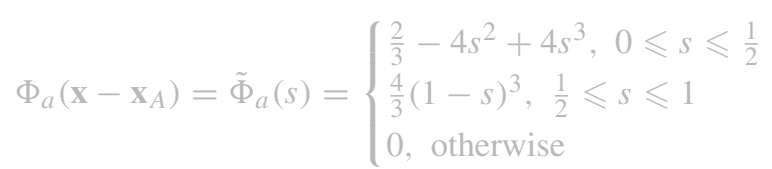

where

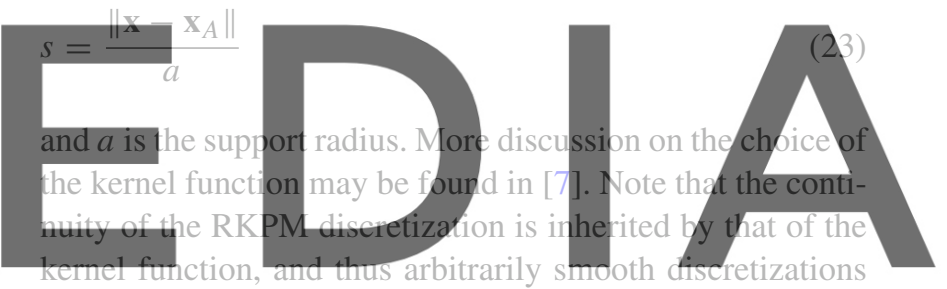

that are independent of the order of approximation may be

dowinkoaduche version without the watermark The discrete RKPM solution $u^{h}(\mathbf{x})$ is given by

$u^{h}(\mathbf{x})=\sum_{A=1}^{\mathrm{n}_{\mathrm{np}}} \Psi_{A}(\mathbf{x}) u_{A}$

where $u_{A}$ 's are the unknown DOFs associated with Lagrangian particles. As in the case of NURBS-based IGA, RKPM basis functions are generally non-interpolatory at the Lagrangian-particle locations.

\subsection{Domain integration for RKPM}

As shown in the previous section, the RKPM basis functions are constructed directly in the physical domain without the requirement of an underlying mesh and the corresponding data structures. While this flexibility makes RKPM attractive for approximating large deformation and fragmentation of the material, it also leads to challenges associated with domain integration. The fact that the RKPM basis functions are non-polynomial [see Eq. (18) for their structure] leads to additional challenges for domain integration. 
Domain integration in RKPM is usually carried out using either Gaussian quadrature or nodal integration. To carry out Gaussian quadrature one needs to generate a background mesh, which, in some sense, goes against the philosophy of Meshfree methods. In addition, in order to reap the full benefits of Gaussian quadrature, the integration cells need to be aligned with the supports of the RKPM basis functions, which is not easily accomplished.

On the other hand, pure nodal integration, despite its efficiency, exhibits low convergence rates and rank instability [27]. Special techniques have been developed for RKPM in recent years to circumvent these issues. These include Stabilized Conforming Nodal Integration (SCNI) [28], Stabilized Non-Conforming Nodal Integration (SNNI) [29], Variationally Consistent Integration (VCI) [28,30,31], and Naturally Stabilized Nodal Integration (NSNI) with VCI correction [32] techniques, among others. The main idea of the SCNI technique is to "smooth" the basis-function gradient over conforming integration cells that partition the problem domain. The smoothed gradient $\tilde{\nabla}$ in each integration cell $\Omega_{A}$ is computed using the divergence theorem as follows
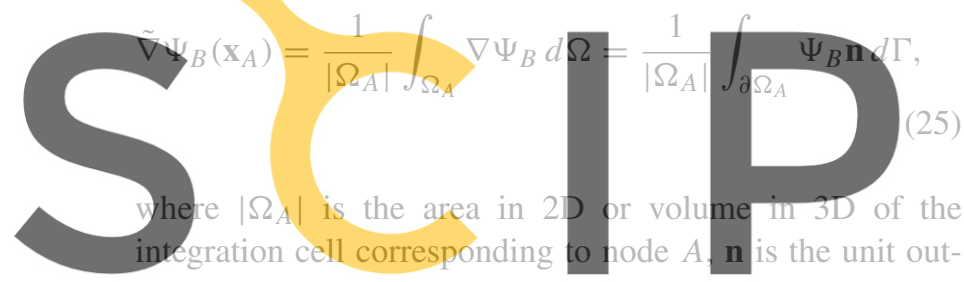

ward normal to the cell boundary $\partial \Omega_{A}$, and index $B$ points to all RKPM basis functions that are supported in $\Omega_{A}$. Register for free at https. WWWW scipedia comption the so-called integration constraint, which ensures that the patch tests are satisfied. Strain smoothing also avoids taking direct derivatives at the Lagrangian nodes. (Doing so in the Lagrangian setting yields instability in the Galerkin solution due to severe underestimation of the strain energy of short-wavelength modes.) While SCNI preserves first-order exactness, for problems involving very large deformation or fragmentation the requirement of conforming cells is challenging to maintain because these need to be periodically regenerated during the simulation. For these reasons, SNNI was introduced in [29], and presents a simplification of SCNI in that the smoothing zones, which are typically simple geometric shapes, are no longer required to be conforming. However, relaxation of the conforming-cell condition can yield non-convergent solutions, because the integration constraint, and thus the linear exactness in the Galerkin solution, are no longer satisfied. Recently, in [32], the authors developed the NSNI technique that overcomes the instabilities of nodal integration by introducing a first-order Taylor expansion of the strains in the internal virtual-work terms. This approach introduces higher-order derivatives in the for- mulation, which may be accommodated in the RKPM and IGA frameworks since both employ smooth discretizations. An implicit gradient [33] has been introduced in NSNI to avoid taking higher-order derivatives of the shape functions.

\section{Numerical aspects of IGA-RKPM coupling}

In this section we focus on the numerical aspects of air-blast FSI that specifically pertain to the IGA-RKPM coupling. We repeat the semi-discrete formulation of the coupled problem from Part I of this paper for convenience:

Find $\mathbf{Y}^{h} \in \mathcal{S}^{h}$, such that $\forall \mathbf{W}^{h} \in \mathcal{V}^{h}$,
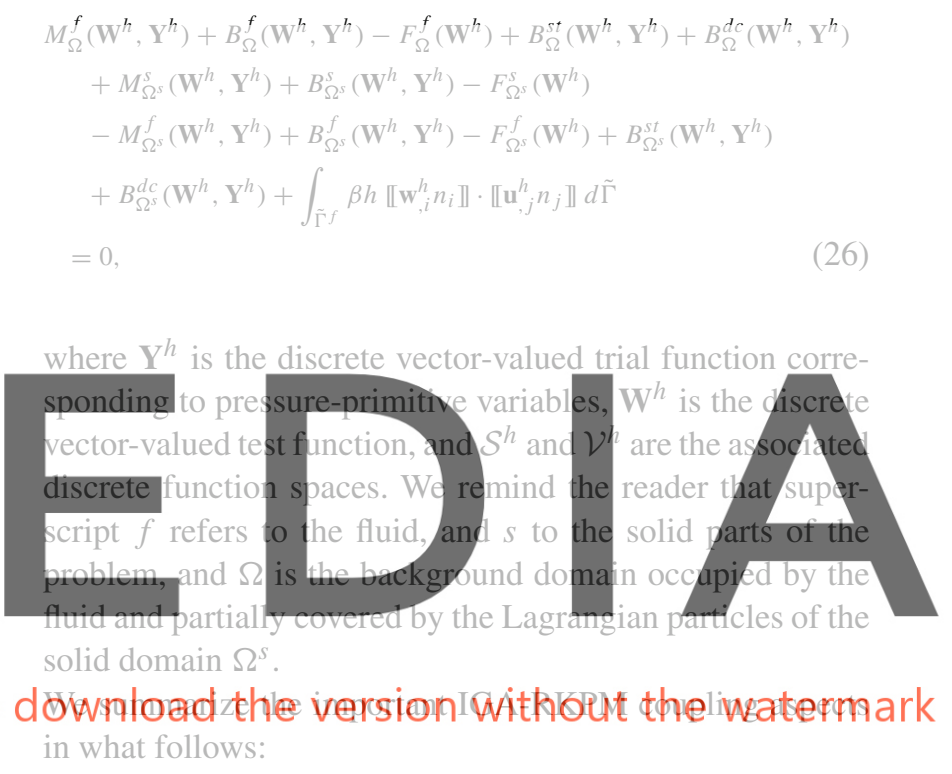

- The spaces of discrete trial and test functions are defined on the background domain $\Omega$, resulting in the FSI problem DOFs residing on the background mesh. Equal-order NURBS discretization is employed for all the unknowns in the variable set $\mathbf{Y}^{h}$.

- The terms on the first line of Eq. (26) are evaluated over the entire background NURBS domain using Gaussian quadrature with $p+1$ points in each tensor-product direction. More efficient quadrature rules are also possible (see [22,23] for recent advances in numerical integration for IGA).

- The terms on the second and third lines of Eq. (26) are evaluated using RKPM domain-integration techniques. Nodal integration is performed using the RKPM Lagrangian points with two options for the background NURBS basis-function gradient evaluation: 1. Direct evaluation at the location corresponding to that of the Lagrangian particle; 2. Smoothed definition as per Eq. (25) with the smoothing zone of simple shape con- 
structed around each Lagrangian particle in the spirit of SNNI. We found that Option 1 is more economical and delivers stable results in the present setting. We are, however, aware of the potential instabilities and inaccuracies associated with this type of integration, and plan to explore an NSNI-type approach in the future work.

- The terms on the fourth line, which correspond to ghostvelocity stabilization [34], are identically zero because the basis functions employed in our discretization are smooth. In principle, the generalization of ghost penalties to higher-order bases involves jumps of higher derivatives, leaving nonzero terms even for spline spaces of maximal continuity. (See, e.g., Remark 1 in [35] or Eq. (11) in [36].) Although we are aware of this generalization, we did not find penalization of jumps in higher derivatives necessary in the present framework.

- Domain integration of the terms on the second and third lines of Eq. (26) require the evaluation of the determinant of the deformation gradient from the solid reference to its current configuration at a Lagrangian-node location $\mathbf{X}_{A}$, which is the nodal quadrature point. In this work the deformation gradient is

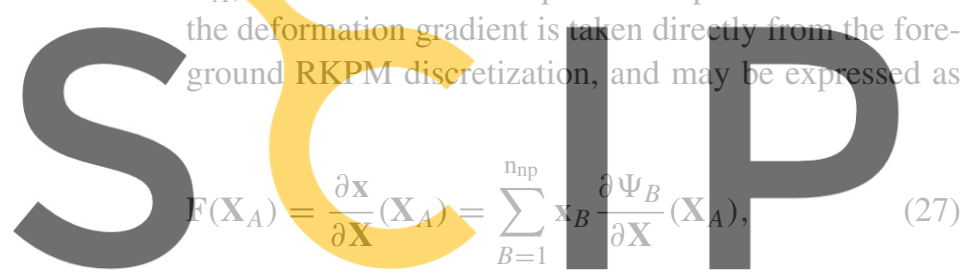

where the above sum is performed only over the RKPM

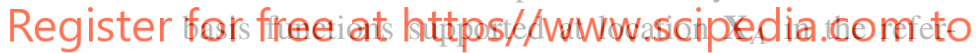
ence configuration. Note that only the current position of the Lagrangian particle $\mathbf{x}_{B}$ and the RKPM basisfunction gradient with respect to the spatial coordinates of the reference configuration $\mathbf{X}$ are required to evaluate the above expression. In our computations, the RKPM basis-function gradient is replaced by its smoothed counterpart over the integration cell in the reference configuration as per Eq. (25), and is computed only once in the beginning of the simulation for efficiency.

As an alternative approach, the determinant of the deformation gradient may be computed from the generating equation for $\mathbf{F}$ (see, e.g., [37])

$$
\left.\frac{\partial \mathbf{F}}{\partial t}\right|_{\mathbf{X}}=\nabla \mathbf{u} \mathbf{F}
$$

or for the determinant itself,

$$
\left.\frac{\partial J}{\partial t}\right|_{\mathbf{X}}=\nabla \cdot \mathbf{u} J
$$

Definitions in Eqs. (28) and (29) do not rely on the discretization of the solid domain and may be better suited when fragmentation scenarios are simulated.

- Stress update described in Part I of this paper is performed at the Lagrangian nodes of the foreground discretization where the history variables are stored. If damage is included in the modeling, which is not the case in the present paper, the corresponding history variables would also be stored at the same locations.

\section{Numerical examples}

In this section we present one $1 \mathrm{D}$ and five 2D computational examples that demonstrate the capabilities, robustness, and accuracy of IGA-RKPM coupling for air-blast FSI. The computational examples are: Sod shock tube, Sedov blast, Taylor bar impact, chamber detonation, flexible panel subjected to a shock load, and detonation with multiple objects. The first three examples test the accuracy of standalone compressibleflow and solid mechanics formulations. Examples four and five are the same as in Part I of the present paper. The

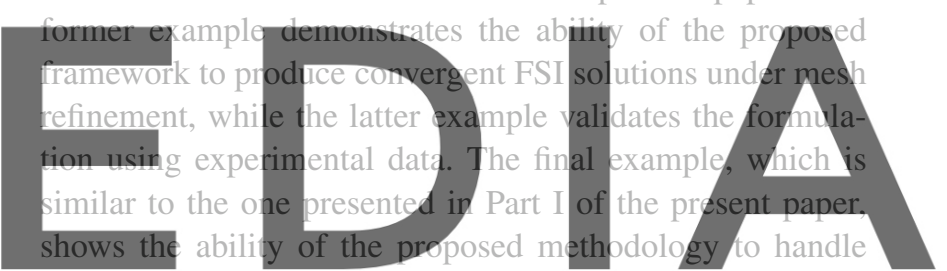

blast in the presence of multiple objects without restriction on their motion and including contact between the objects. dewnload the versien withd Qut the waternark

linear consistency and kernel given by Eq. (22) are employed in all computations. Unless otherwise stated, in all computations the fluid is assumed to have properties of air with constant viscosity $\mu=1.81 \times 10^{-5} \mathrm{~kg} /(\mathrm{m} \mathrm{s})$, Prandtl number 0.72 , and adiabatic index $\gamma=1.4$. The time step for each problem is selected from considerations of stability and two-to-four explicit corrector passes are employed in the computations.

\subsection{Sod shock tube problem}

We compute a 1D Sod shock tube problem [38], which is an inviscid hydrodynamics example. At the initial time, two material states are prescribed on each half of a unit-length domain. As time evolves, a rarefaction wave, contact discontinuity and shock discontinuity are formed in the domain. On the left, the initial conditions are $\rho=1.0, v=0.0$, and $p=1.0$, while on the right, the initial conditions are $\rho=0.1, v=0.0$, and $p=0.125$. (The problem is specified in non-dimensional units.) The discretization consists of 300 uniform elements, and the numerical results at time $t=0.2$ 


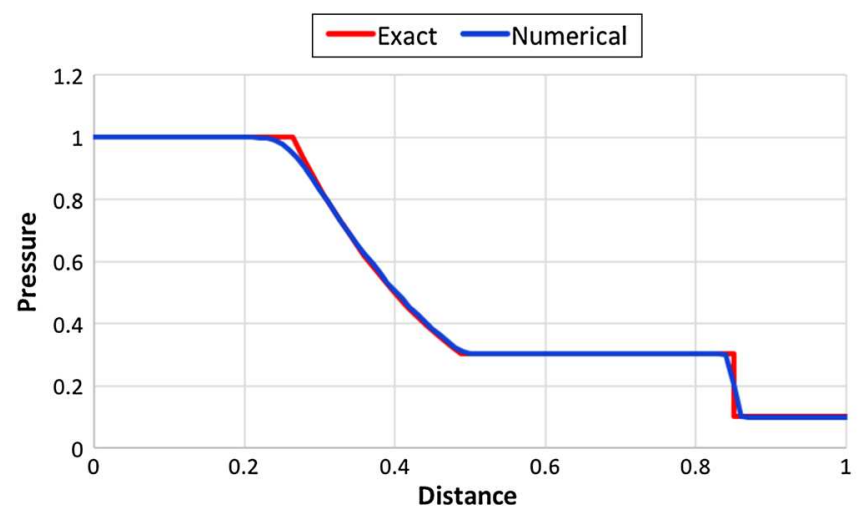

(a)

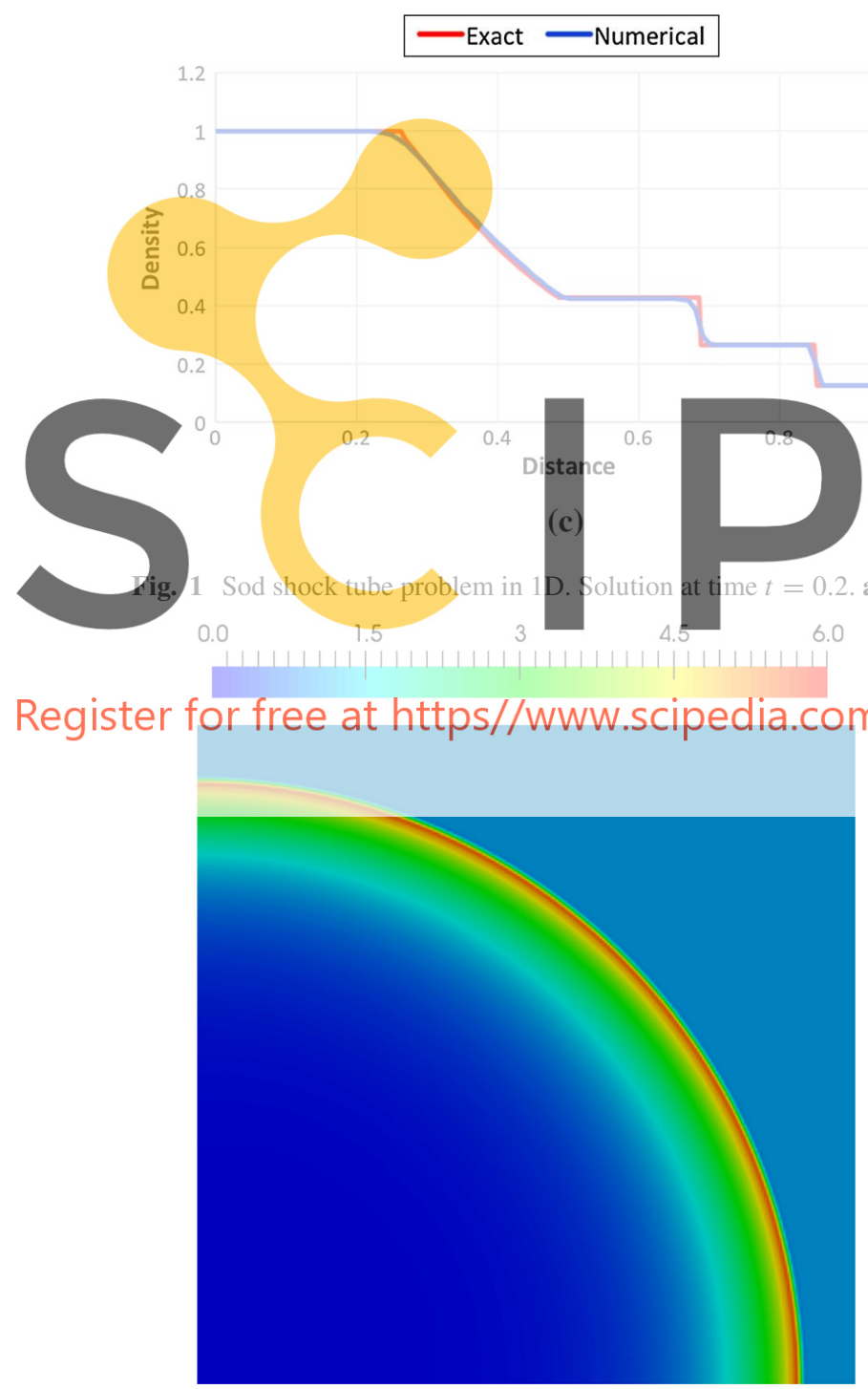

Fig. 2 Sedov blast problem in 2D. Density contours at time $t=1.0$. (Color figure online)

are compared to the analytical solution in Fig. 1. As can be seen in the figure, the numerical results have no oscillations, and match the exact solution very well.

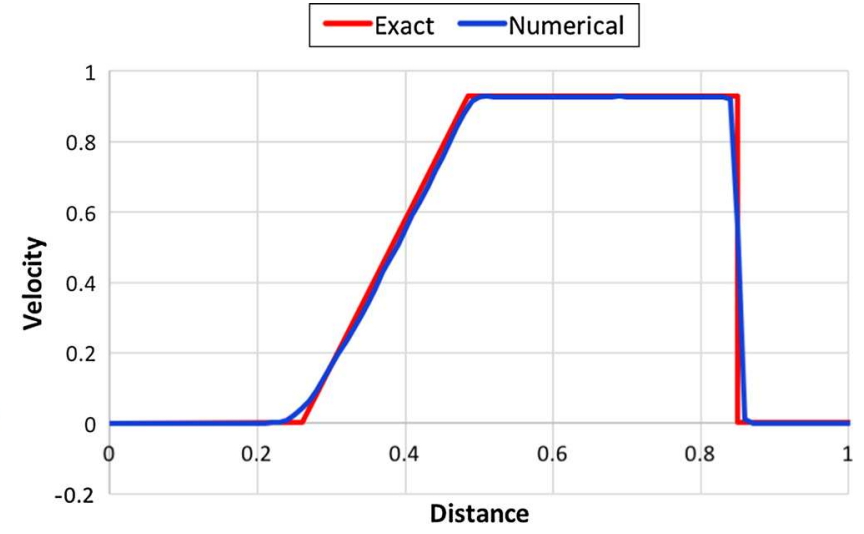

(b)

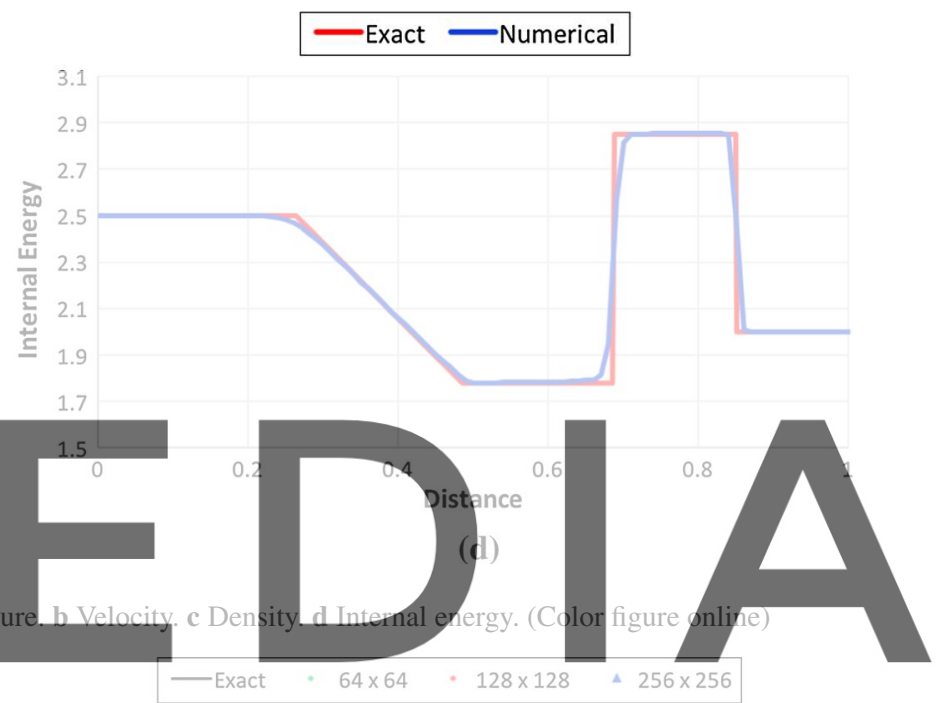

download the version without the watermark

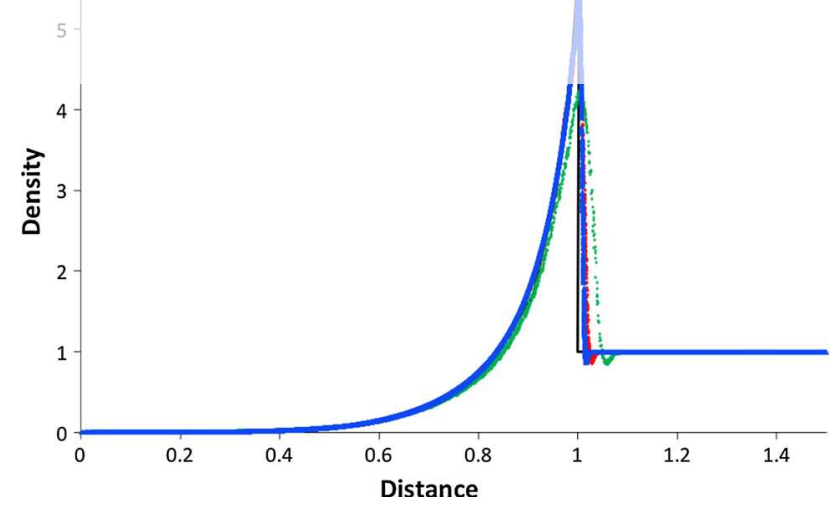

Fig. 3 Sedov blast problem in 2D. Scatter plot of density versus radial coordinate for the three meshes employed. The exact solution is also plotted to illustrate convergence with mesh refinement. (Color figure online)

\subsection{Sedov blast problem}

We compute the 2D inviscid Sedov blast problem [39] on a square domain with edge length $L=1.1$. A sudden release of energy at the origin creates an expanding shock 
to 0.25 . (The problem is also specified in non-dimensional

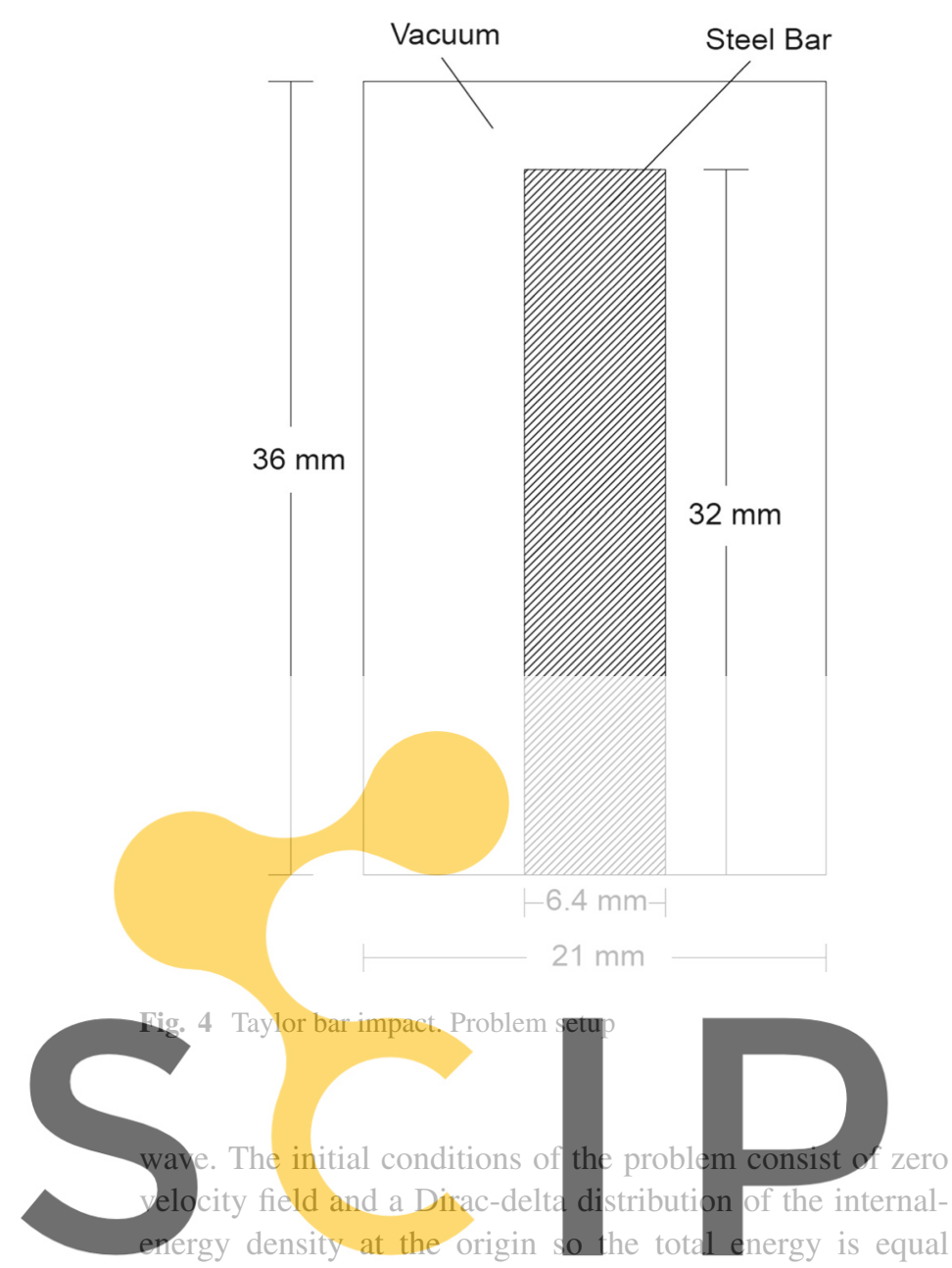

units.) The initial density is set to $\rho=1$, the temperature is set consistent with the internal energy density, and pressure is set consistent with the ideal gas law. Meshes of $64 \times 64,128 \times 128$, and $256 \times 256$ elements are employed in the computations. Figure 2 shows the density contours at time $t=1.0$ on the mesh of $256 \times 256$ elements. The solution appears to be smooth, stable, and radially symmetric. Figure 3 shows the scatter plot of density vs. distance from the origin at time $t=1.0$ for all three meshes. Convergence to the analytical result is evident from the plot. Very little scatter in the data is also observed, suggesting the methodology has excellent symmetry preservation properties.

\subsection{Taylor bar impact}

We simulate a steel bar impacting a rigid wall at the initial velocity of $227 \mathrm{~m} / \mathrm{s}$. The bar has an initial height of $32 \mathrm{~mm}$, width of $6.4 \mathrm{~mm}$, and is discretized using $250 \times 150$ particles. The background domain has a height of $36 \mathrm{~mm}$, width of $21 \mathrm{~mm}$, and is discretized using $77 \times 45$ elements.

No-penetration and zero tangential-stress boundary conditions are applied at the bottom wall. The bar is also assumed

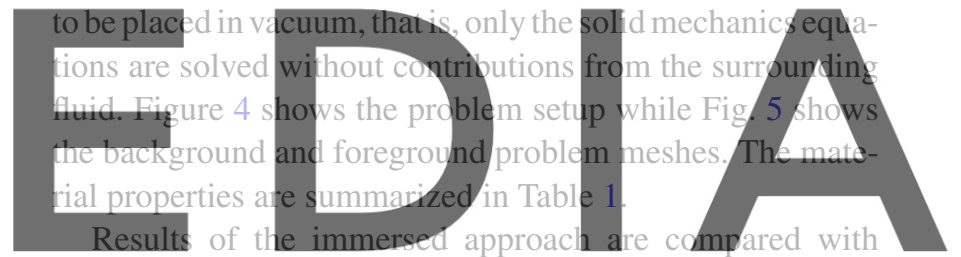

those obtained by solving the problem using a Lagrangian

Register for free at https//www.scipedia.com to download the version without the watermark Fig. 5 Taylor bar impact. Background and foreground problem meshes
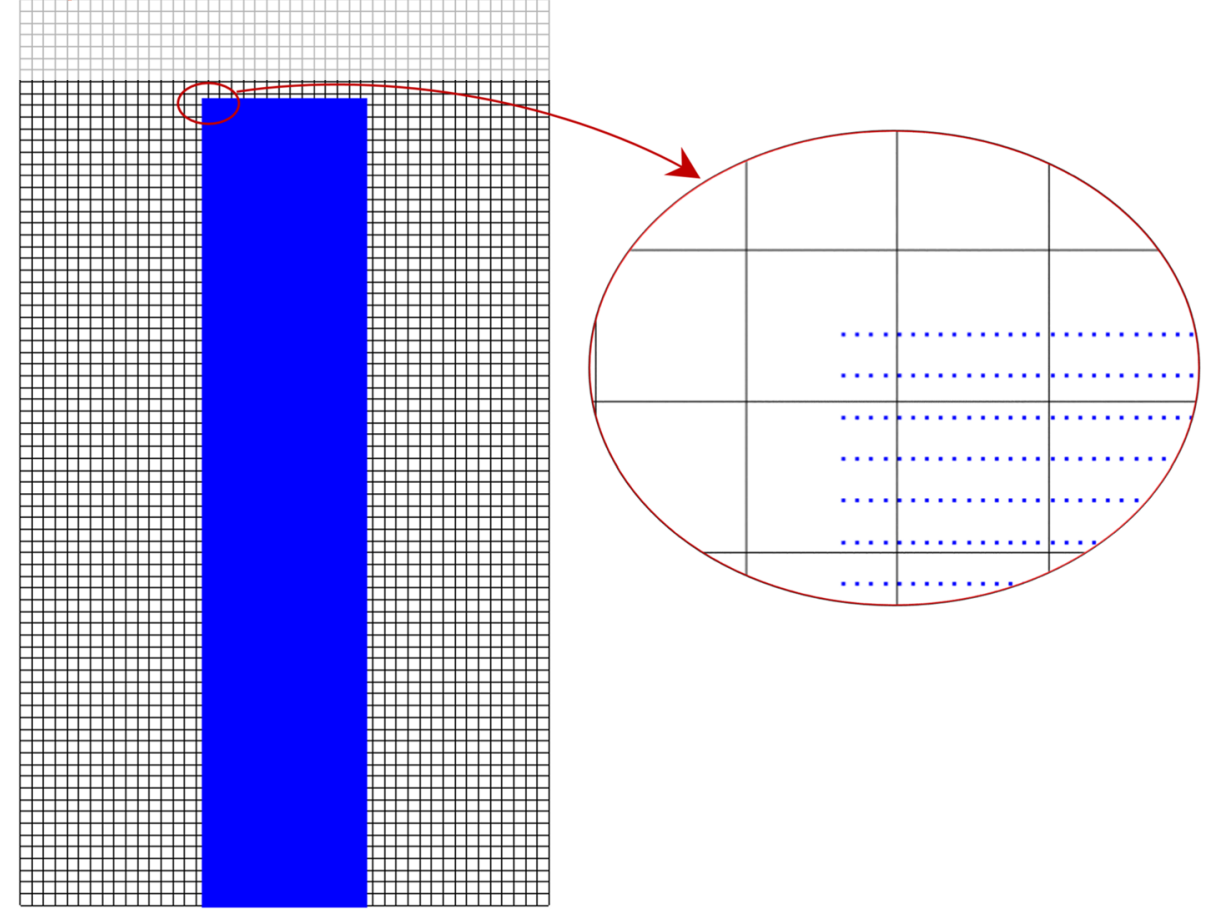
Table 1 Taylor bar impact

\begin{tabular}{ll}
\hline Young's modulus E & $200 \mathrm{GPa}$ \\
Poisson's ratio $v$ & 0.30 \\
Density $\rho$ & $2700 \mathrm{~kg} / \mathrm{m}^{3}$ \\
Yield stress $\sigma_{y}$ & $0.29 \mathrm{GPa}$ \\
Hardening modulus h & $0.1 \mathrm{GPa}$ \\
\hline
\end{tabular}

Material properties

RKPM formulation. The Taylor-bar height and width time histories are plotted in Fig. 6. Excellent agreement between the two computations is achieved for these quantities. Figure 7 shows the final deformed shape of the Taylor bar with a zoom on the impact region where most of the deformation occurs. The immersed and RKPM computations are in very good agreement with each other, demonstrating that the immersed methodology is capable of accurately capturing the solid plastic deformations.

\subsection{Chamber detonation}

In this coupled FSI example, a steel bar is subjected to a detonation blast load. A bar with dimensions $0.2 \mathrm{~m} \times$ $0.1 \mathrm{~m}$ is placed at the center of a closed chamber with dimensions $0.4 \mathrm{~m} \times 0.4 \mathrm{~m}$. The bar thickness is set to $3.5 \mathrm{~mm}$. Figure 8 shows the problem description. The bar material properties correspond to those of steel, and are summarized in Table 2. The air in the chamber is initially at rest with $T=270 \mathrm{~K}$ and $p=100,000 \mathrm{~Pa}$. The detonation is initiated by setting higher-than-ambient values of the pressure, $p=6,746,268.65 \mathrm{~Pa}$, and temperature, $T=1,465 \mathrm{~K}$, in a semi-circular region centered on the
Fig. 6 Taylor bar impact.

Comparison of the time history

of Taylor bar height (a) and

width (b) between the immersed

and Lagrangian RKPM

simulations. (Color figure
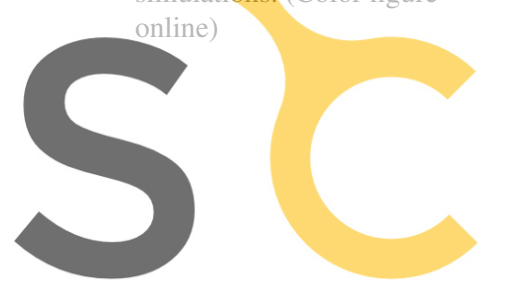

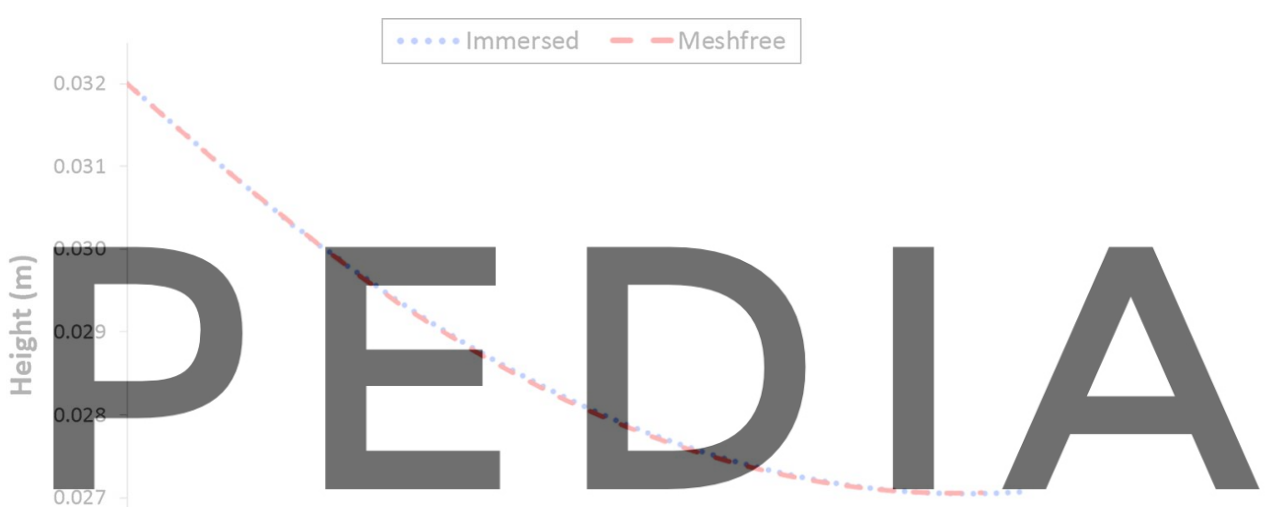

Register for free at https//www.scipedia.com to download the version without the waterpriark Time (s)

(a)

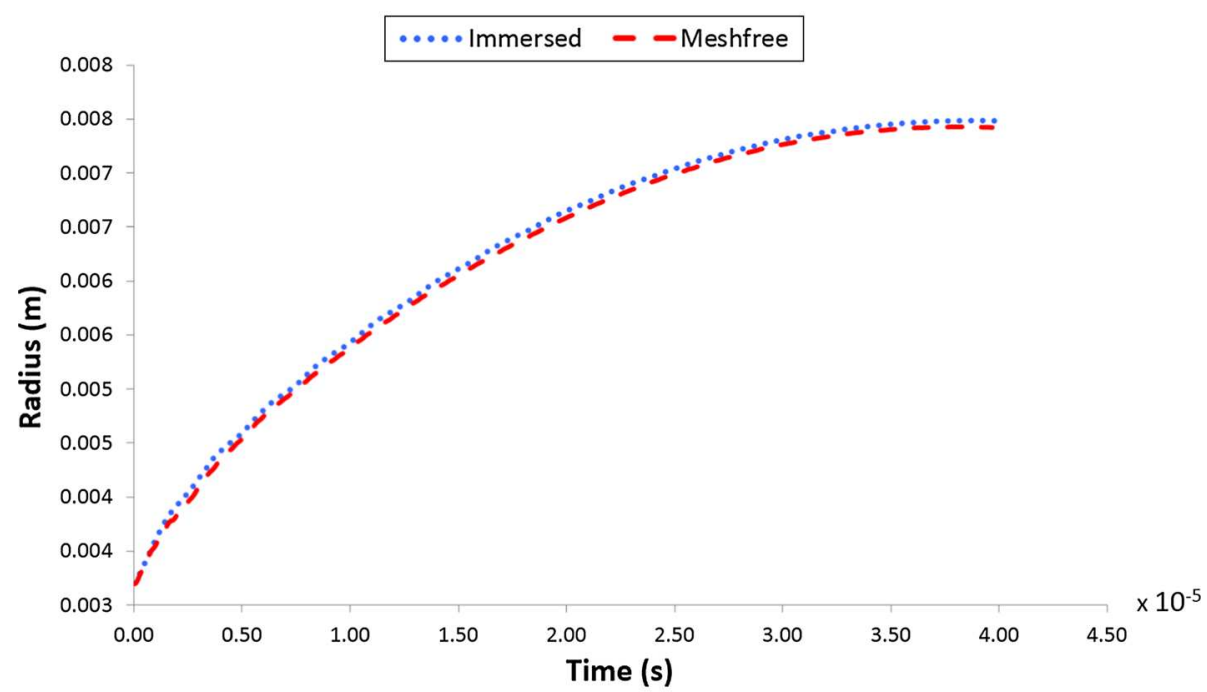

(b) 
Fig. 7 Taylor bar impact. Overlapping, deformed configurations of the Taylor bar from the immersed (blue) and RKPM (red) simulations. Zoom on the region near the impact where most of the deformation occurs. (Color figure online)
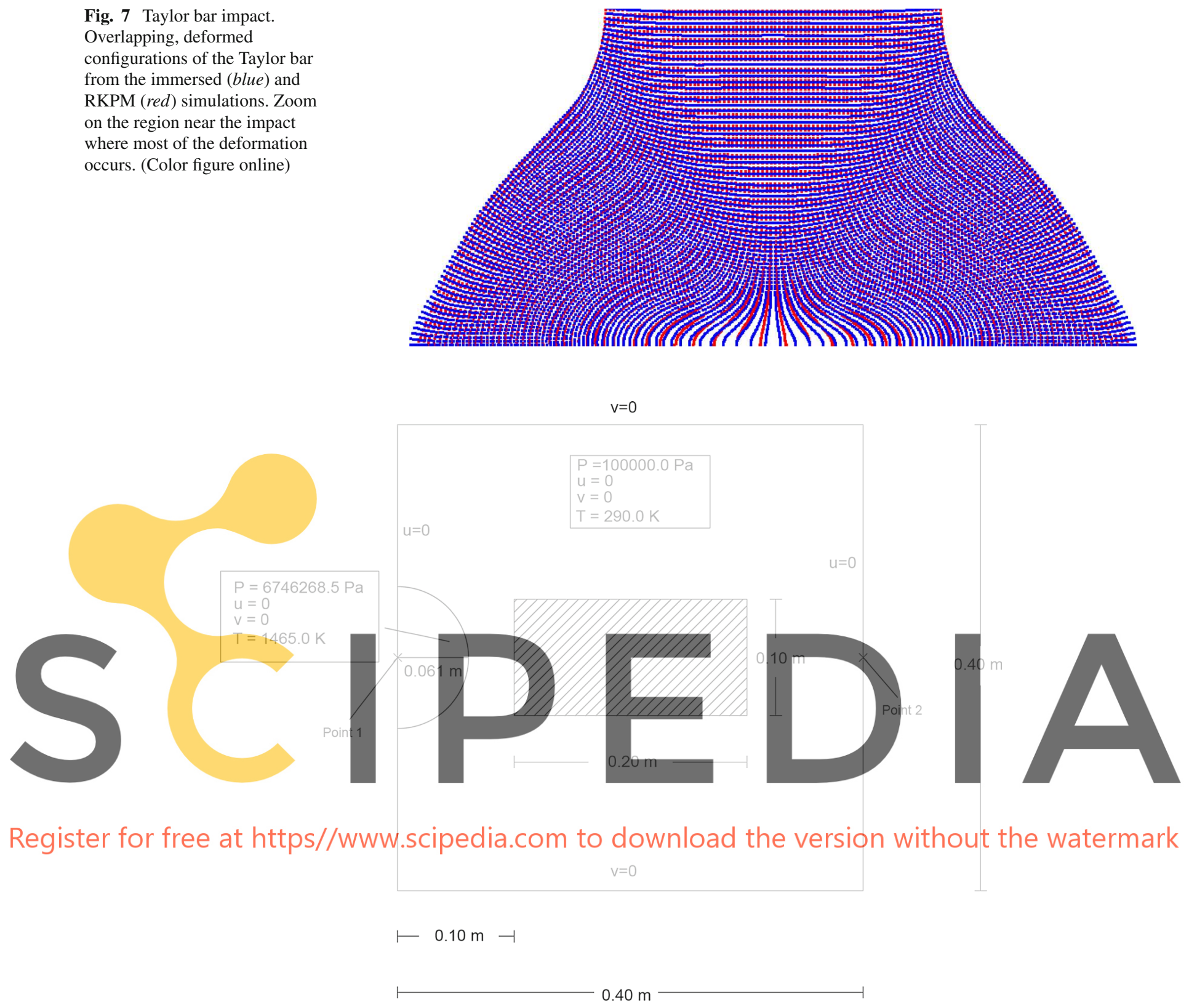

Fig. 8 Chamber detonation. Problem setup and dimensions

Table 2 Chamber detonation

Young's modulus E

$200 \mathrm{GPa}$

Poisson's ratio $v$

0.29

Density $\rho$

$7870 \mathrm{~kg} / \mathrm{m}^{3}$

Yield stress $\sigma_{y}$

$0.4 \mathrm{GPa}$

Hardening modulus $\mathrm{h}$

$0.1 \mathrm{GPa}$

Material properties of a steel bar

left wall and with radius of $6.1 \mathrm{~mm}$. Free-slip and nopenetration boundary conditions are assumed at the chamber walls.
Four problem discretizations with increasing mesh refinement levels are considered: (1) Fluid: $40 \times 40$ elements; Solid: $53 \times 26$ particles; (2) Fluid: $80 \times 80$ elements; Solid: $105 \times 53$ particles; (3) Fluid: $120 \times 120$ elements; Solid: $158 \times 79$ particles; (4) Fluid: $160 \times 160$ elements; Solid: $210 \times 105$ particles. Figure 9 shows the air pressure at different time instants and the final, deformed shape of the bar computed on the finest mesh. Note the "mushrooming" at the left edge of the bar and very large deformation at the bar corners. Also note the permanent indentation on the right edge of the bar resulting from shock waves bouncing off the right wall and impacting the specimen. 


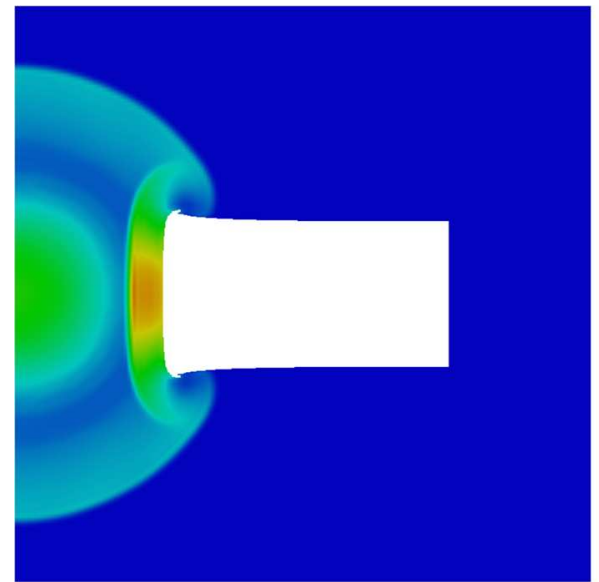

(a)

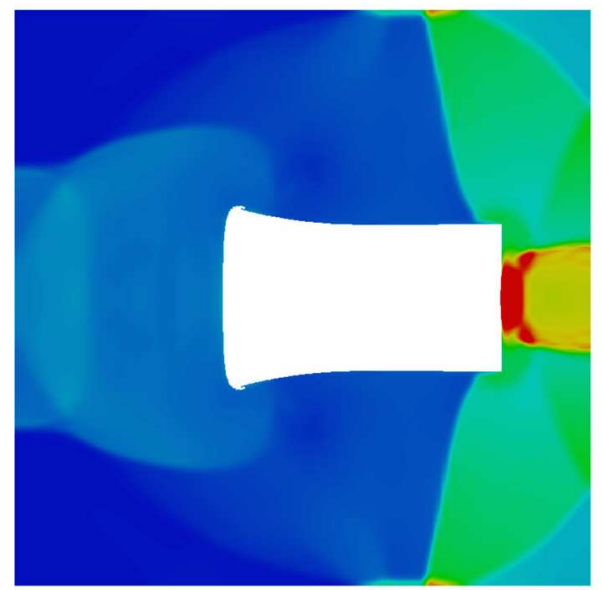

(c)

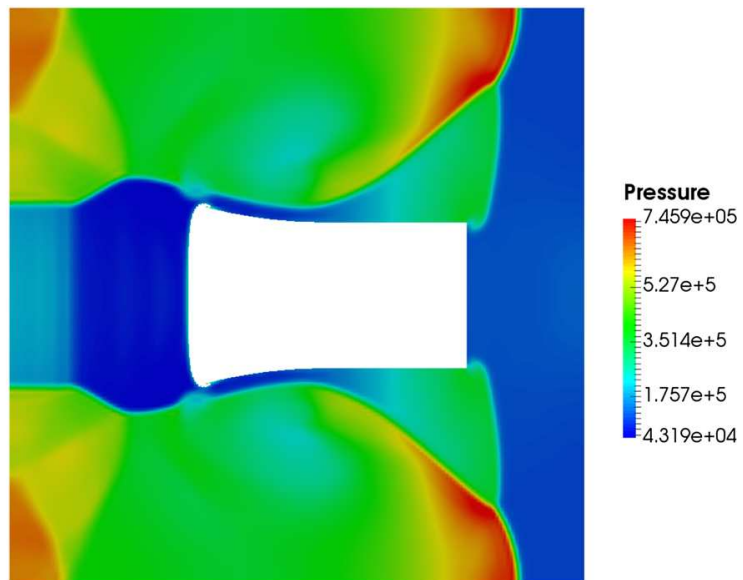

(b)

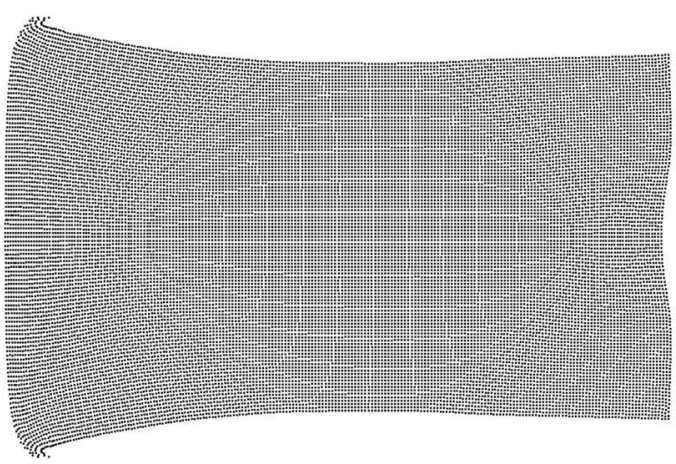

(d)

Fig. 9 Chamber detonation. Pressure at different time instants and final, deformed configuration of a steel bar. a $0.1 \mathrm{~ms}$. b $0.4 \mathrm{~ms}$. c $0.7 \mathrm{~ms}$. d Final configuration. (Color figure online)

Figure 10 shows the time history of the bar center-of-mass displacement, and pressure at the detonation center (Point 1 in the figure) and right-wall center (Point 2 in the figure). The results exhibit good convergence with mesh refinement, and are in good agreement with the ALE results from Part I of this paper. Shock waves bouncing between the right wall and moving bar are captured very well in the simulations.

\subsection{Shock wave impacting an elastic panel}

In this example, a thin steel panel is impacted by a planar shock wave in air. The problem setup is shown in Fig. 11. The panel thickness is $1 \mathrm{~mm}$, and it extends $40 \mathrm{~mm}$ from a mounting point with forward-facing step geometry where it is clamped. The fluid domain has dimensions $600 \mathrm{~mm} \times 80 \mathrm{~mm}$. The step has a height of $15 \mathrm{~mm}$ and starts $335 \mathrm{~mm}$ from the left boundary. Inflow boundary conditions are applied on the left side while rigid-wall boundary conditions are applied elsewhere. In this example the panel material is assumed to be elastic with the properties given in Table 3. The shock is placed at $330 \mathrm{~mm}$ from the left wall and travels into air at rest with density $\rho=1.2 \mathrm{~kg} / \mathrm{m}^{3}$ and pressure $p=100 \mathrm{kPa}$. Behind the shock the density is $\rho=1.6458 \mathrm{~kg} / \mathrm{m}^{3}$, the pressure is $p=156.18 \mathrm{kPa}$, and the horizontal velocity $v=112.61 \mathrm{~m} / \mathrm{s}$. The background mesh has $480 \times 64$ elements, while the panel foreground mesh makes use of $10 \times 500$ Lagrangian particles. The step is modeled in an immersed fashion with zero velocity assigned to the block of control points corresponding to the step location. The problem has been previously investigated both experimentally and numerically in $[40,41]$, and is also computed in Part I of this paper.

Figure 12 shows the pressure field and panel displaced configuration at different time instants. After the initial impact of the shock wave the panel begins to oscillate. Fig- 


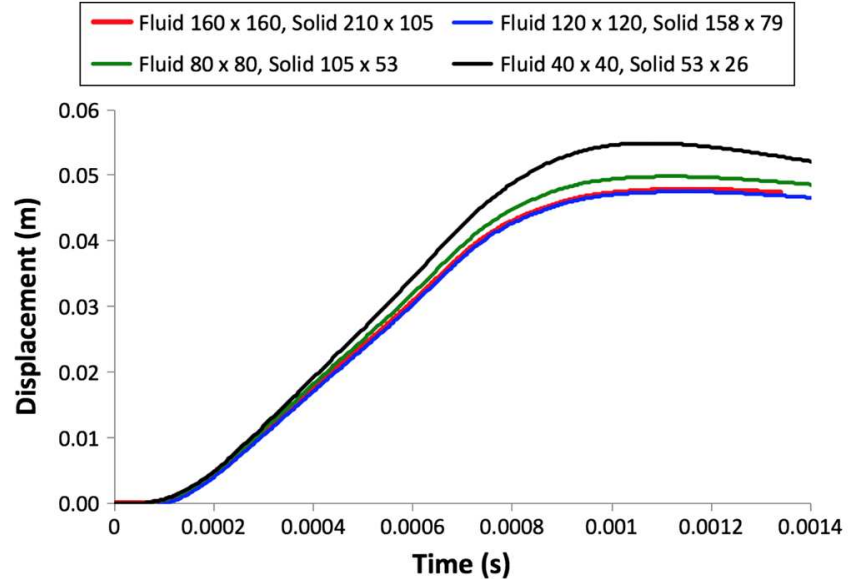

(a)

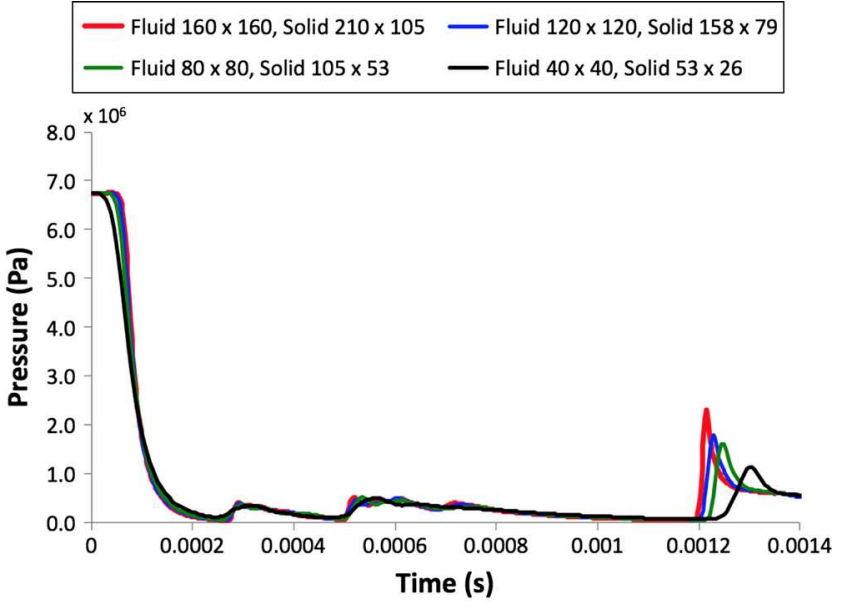

(b)

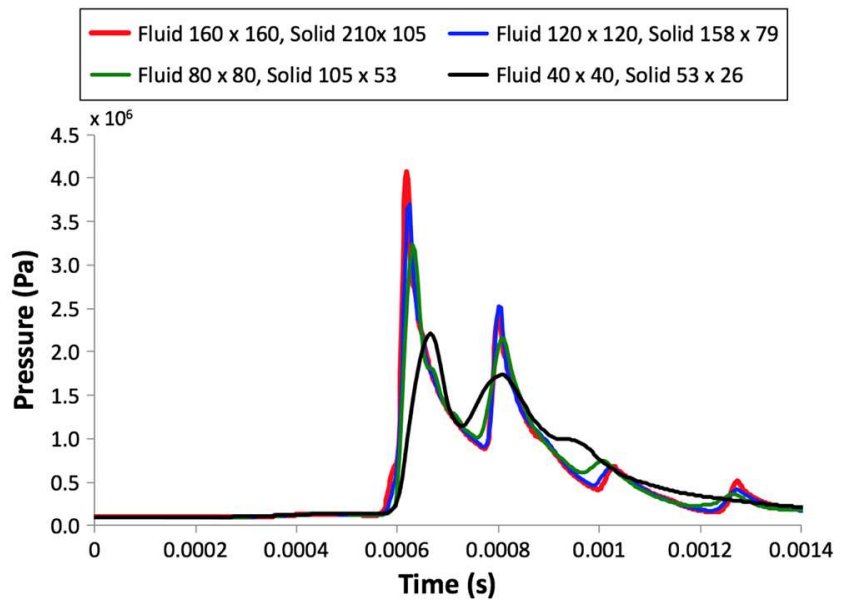

(c)

Fig. 10 Chamber detonation. a Horizontal displacement of the bar center of mass; $\mathbf{b}$ pressure at the center of detonation; $\mathbf{c}$ pressure at the center of the right wall. (Color figure online)

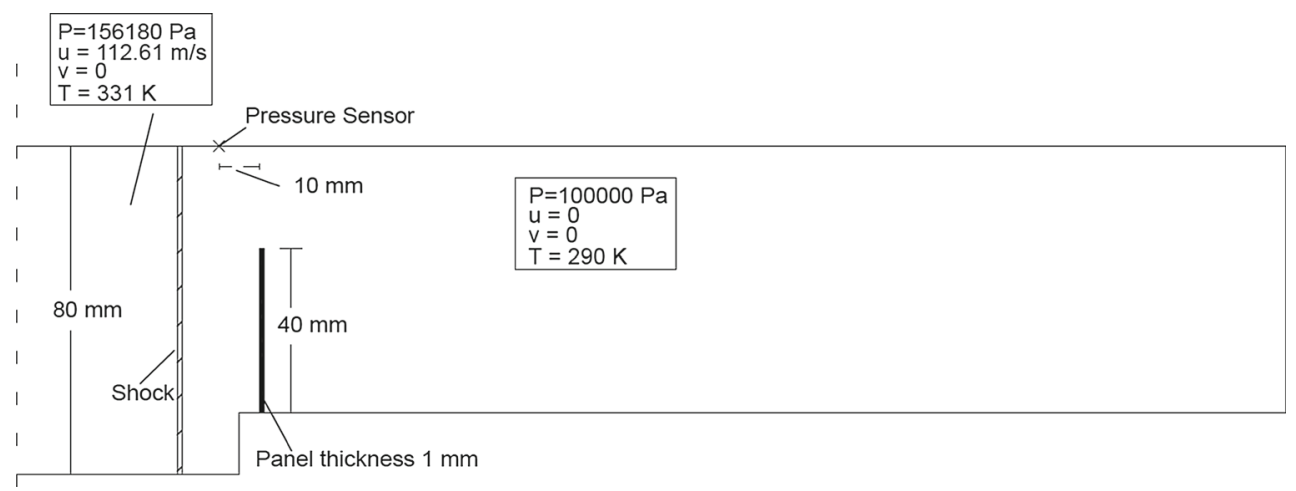

Fig. 11 Shock wave impacting an elastic panel. Problem setup 
Table 3 Shock wave impacting an elastic panel

Young's modulus E

$220 \mathrm{GPa}$

Poisson's ratio $v$

0.33

Density $\rho$

$7600 \mathrm{~kg} / \mathrm{m}^{3}$

Material properties of the steel panel

ure 13a shows a comparison of the computed and measured panel-tip displacement time histories. The two quantities are in good agreement, both in terms of the oscillation magnitude and frequency, suggesting that the background mesh is sufficiently fine to provide the appropriate level of the panel through-thickness resolution. Figure 13b shows a comparison of the computed and measured pressure time histories at the pressure-sensor position. Very good agreement is obtained in this case as well, suggesting that the complex dynamics of shocked flow is well captured in the simulation.

Remark Note that in Part 1 of the paper, toward the end of the simulation, the predicted pressure signal starts to drift from the experimental curve due to a spurious reflection wave arriving from the left boundary where inflow boundary conditions are prescribed. No such drift is seen in the present simulation, because the inflow boundary is placed much further to the left of the panel than in the simulation presented in Part I of the paper.

\subsection{Detonation with multiple objects}

This last example shows the ability of the proposed method to naturally handle scenarios of detonation in the presence of multiple objects. Several rectangular objects are placed in a rectangular chamber with dimensions $1.2 \mathrm{~m} \times 0.8 \mathrm{~m}$, and are subjected to a detonation load. Figure 14 shows the problem setup. The largest objects have dimensions $0.26 \mathrm{~m}$ $\times 0.075 \mathrm{~m}$, the smallest ones $0.0375 \mathrm{~m} \times 0.05 \mathrm{~m}$, and the rest $0.075 \mathrm{~m} \times 0.1 \mathrm{~m}$. The detonation is initiated by assuming air at rest with $T=270 \mathrm{~K}$ and $p=100,000 \mathrm{~Pa}$, and elevating the pressure to $p=$ 11.0 $\mathrm{MPa}$ and temperature to $T=1,550 \mathrm{~K}$ in the zone of radius $0.05 \mathrm{~m}$. All objects are assumed to have the material properties of steel, and are modeled as elastic. Slip boundary conditions are applied at the chamber walls. Uniform mesh with dimension $0.006 \mathrm{~m}$ for the air and $0.0034 \mathrm{~m}$ for the solid objects is employed for the problem discretization.

Figure 15 shows the air speed and the solid deformed configuration at different instants after the detonation is initiated. The solid objects contact each other, impact the chamber walls, and, in general, move without any restrictions under the action of blast waves. The flow solution remains stable with crisp resolution of the shock waves throughout the computation.

1.2105

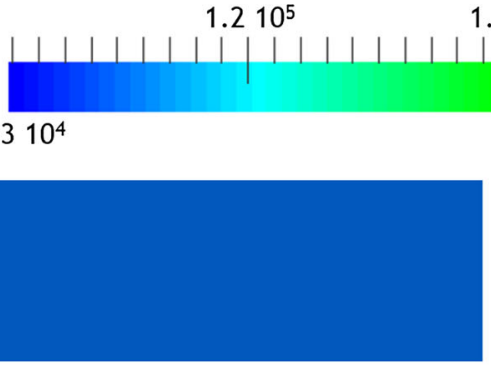

(a)

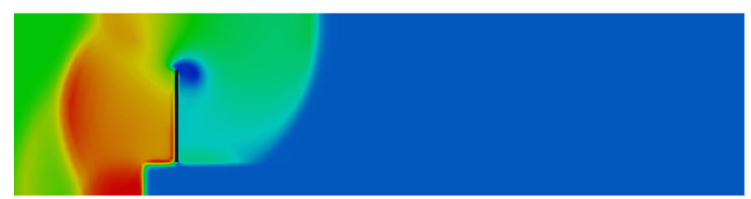

(c)

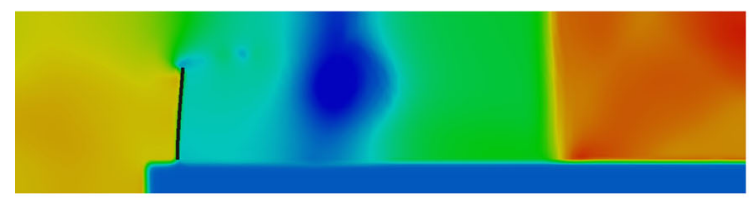

(e)

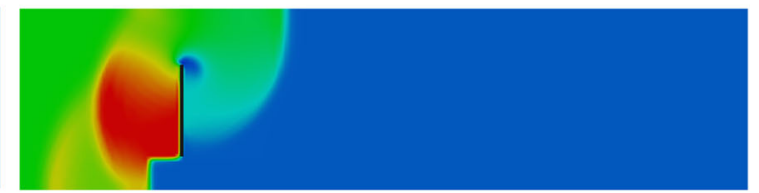

(b)

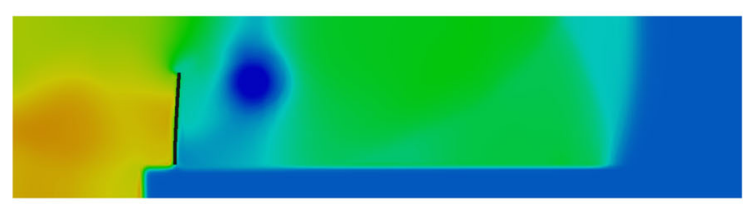

(d)

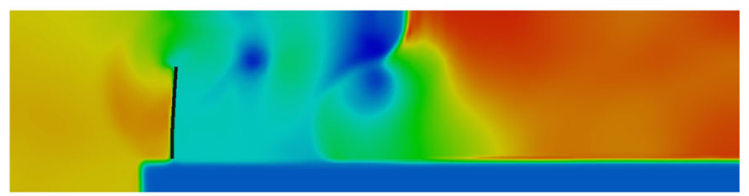

(f)

Fig. 12 Shock wave impacting an elastic panel. Pressure field and deflected panel at different time instants. a $70 \mu$ s. b $150 \mu$ s. c $200 \mu$ s. d $570 \mu$ s. e $1000 \mu$ s. f $1245 \mu$ s. (Color figure online) 


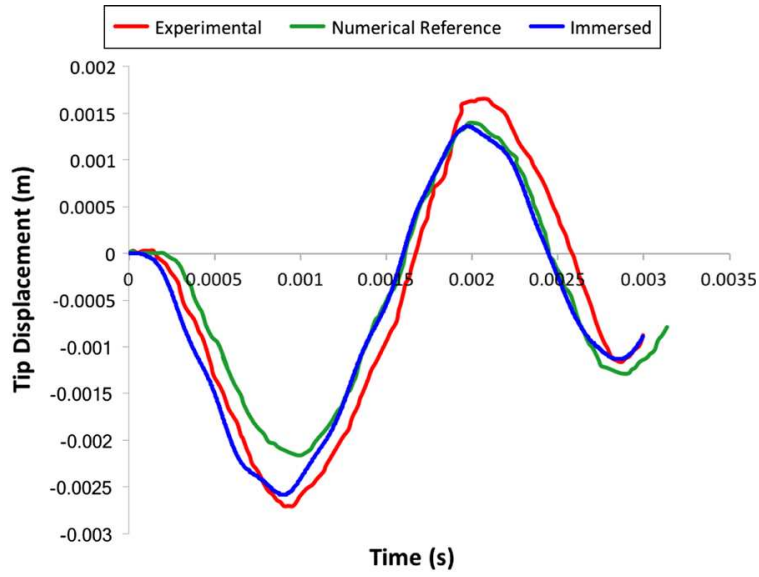

(a)

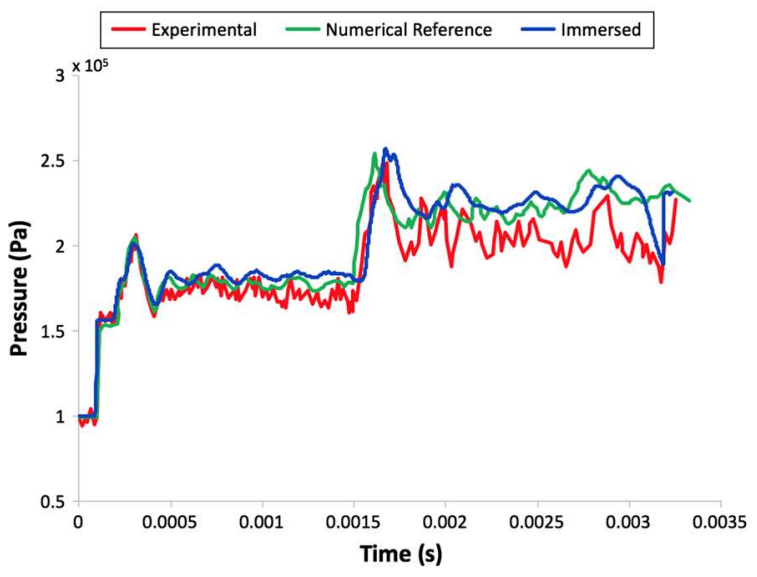

(b)

Fig. 13 Shock wave impacting an elastic panel. Time history of a panel tip displacement; b pressure at the sensor location. Computational results from [41] are also shown for comparison. (Color figure online)

\section{Conclusions}

In Part II of this paper, a computational framework for airblast FSI based on an immersed approach, which models the interaction of compressible flow in the high-Mach-number regime with inelastic solids and structures, is discretized using the IGA-RKPM coupling. The NURBS-based isogeometric background discretization is fixed and provides the discrete trial and test function spaces for the coupled FSI problem. The RKPM-based foreground discretization is moving with the solid material particles and is employed to track its current position, store history-dependent variables, and perform numerical quadrature.

Several attributes of the coupled IGA-RKPM formulation are exploited to improve the accuracy and robustness of the immersed air-blast FSI framework. Most notably, the higher-order accuracy and smoothness of the background discretization delivers high-quality compressible-flow solutions with shocks. In addition, the higher-order smoothness of the background basis functions gives a continuous representation of the strain-rate field, which greatly improves the quality of the solid mechanics solution. Finally, the ghost velocity stabilization needed for the stability of the $C^{0}$-continuous case is completely obviated.

This work presents first steps in the direction of airblast FSI and defines the underlying framework and core methodologies for this class of problems. Future efforts will be focused on 3D computations, improvement of the nodal domain-integration techniques, and accurate representation of the solid disintegration and fragmentation during blast. In addition, for the solid discretization, it may be beneficial to develop a methodology that directly employs the RKPM approximation for the space of trial functions.
Fig. 14 Detonation with multiple objects. Problem setup

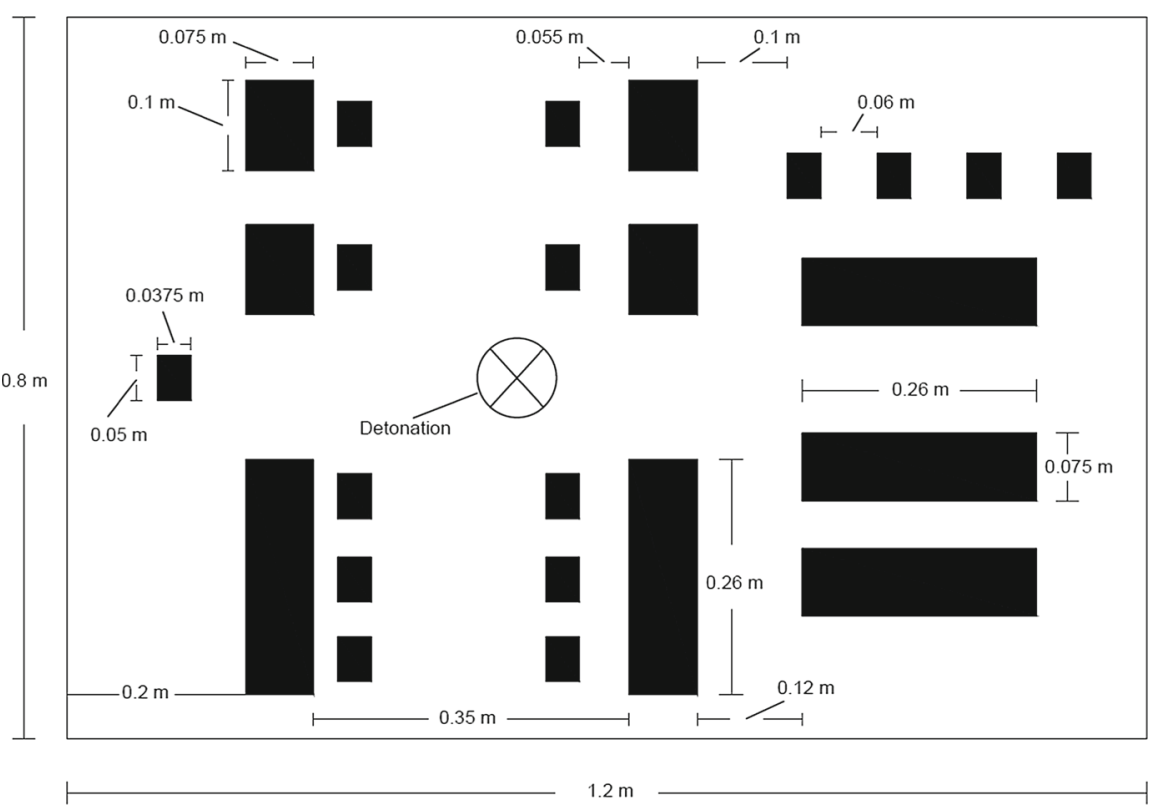




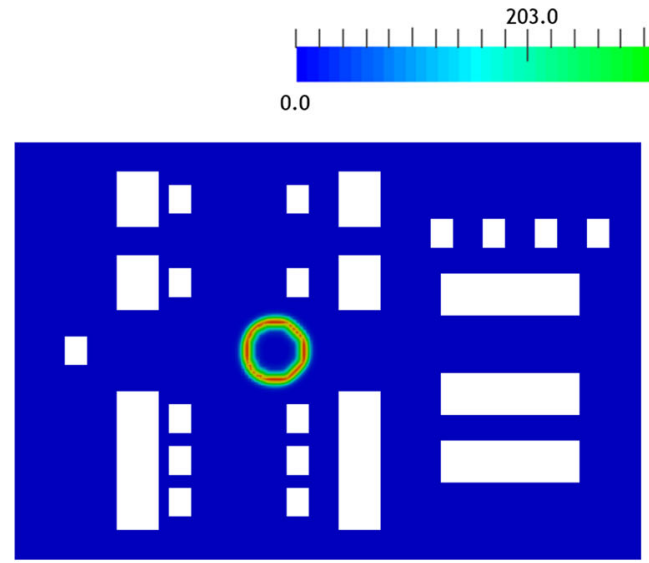

(a)

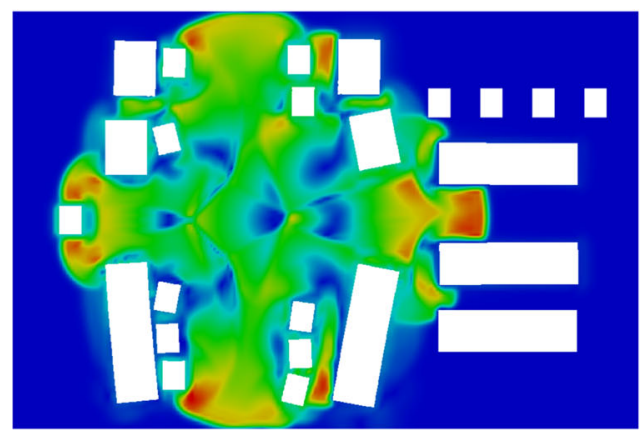

(c)

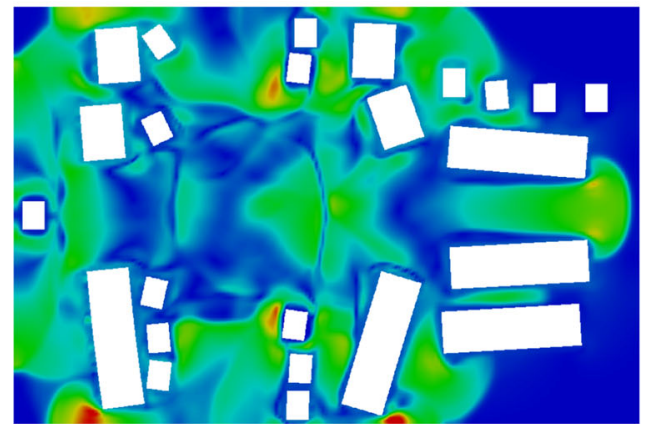

(e)

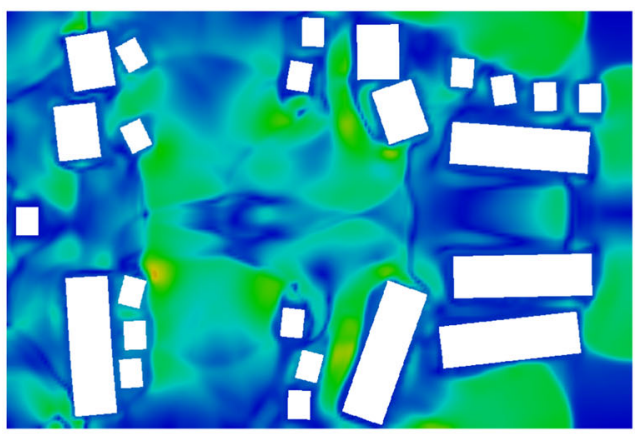

(g)

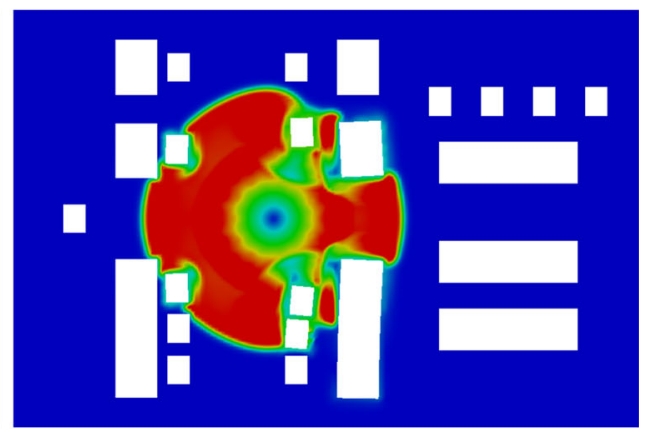

(b)

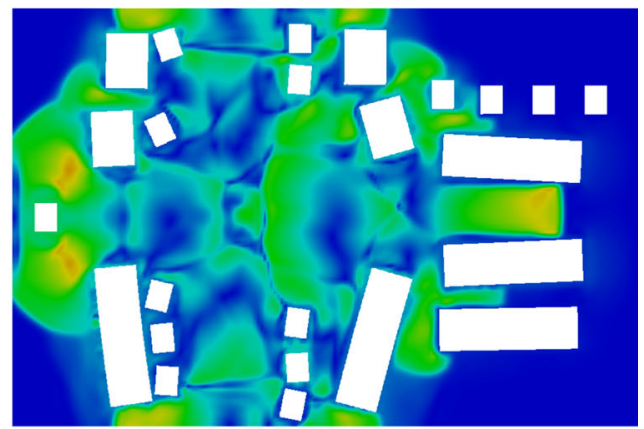

(d)

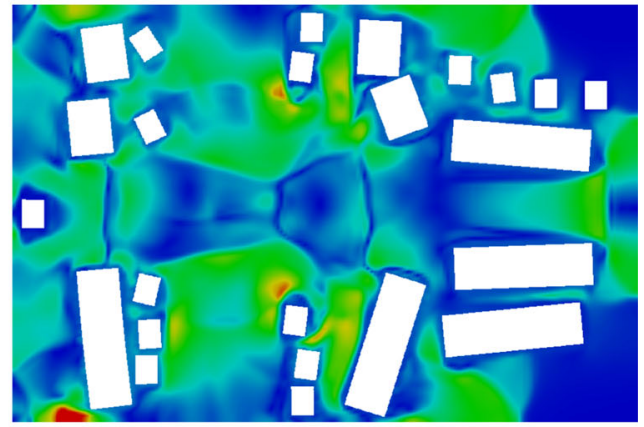

(f)

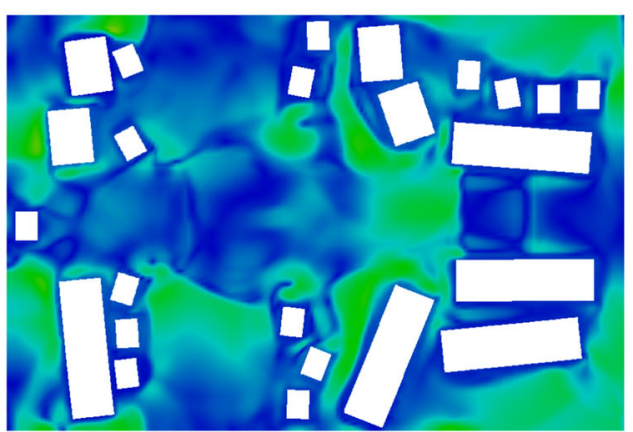

(h)

Fig. 15 Detonation with multiple objects. Air speed and solid in the current configuration at different instants during the simulation. a $0.01 \mathrm{~ms}$. b $0.2 \mathrm{~ms}$. c $0.4 \mathrm{~ms}$. d $0.6 \mathrm{~ms}$. e $0.8 \mathrm{~ms}$. f $1.0 \mathrm{~ms}$. g $1.2 \mathrm{~ms}$. h $1.4 \mathrm{~ms}$. (Color figure online) 
Acknowledgements YB was partially supported by the ARO W911NF14-1-0296 award. DK was supported by AFOSR Award FA9550-121-0046. This support is gratefully acknowledged. The IGA-RKPM coupling was carried out in PetIGA, a software framework that implements NURBS-based IGA [42].

\section{References}

1. Hughes TJR, Cottrell JA, Bazilevs Y (2005) Isogeometric analysis: CAD, finite elements, NURBS, exact geometry, and mesh refinement. Comput Methods Appl Mech Eng 194:4135-4195

2. Cottrell JA, Hughes TJR, Bazilevs Y (2009) Isogeometric analysis. Toward integration of CAD and FEA. Wiley, New York

3. Farin GE (1999) NURBS: from projective geometry to practical use. AK Peters Ltd, Natick

4. Piegl L, Tiller W (2012) The NURBS book. Springer, Berlin

5. Rogers DF (2000) An introduction to NURBS: with historical perspective. Elsevier, Amsterdam

6. Chen JS, Belytschko T (2015) Meshless and meshfree methods. In: Encyclopedia of applied and computational mathematics. Springer, Berlin, pp 886-894

7. Liu WK, Jun S, Zhang YF (1995) Reproducing kernel particle methods. Int J Numer Methods Fluids 20(8-9):1081-1106

8. Liu WK, Jun S, Li S, Adee J, Belytschko T (1995) Reproducing kernel particle methods for structural dynamics. Int J Numer Methods Eng 38(10):1655-1679

9. Chen JS, Pan C, Wu C-T, Liu WK (1996) Reproducing kernel particle methods for large deformation analysis of non-linear structures. Comput Methods Appl Mech Eng 139(1):195-227

10. Bazilevs Y, Calo VM, Cottrell JA, Hughes TJR, Reali A, Scovazzi G (2007) Variational multiscale residual-based turbulence modeling for large eddy simulation of incompressible flows. Comput Methods Appl Mech Eng 197:173-201

11. Cottrell JA, Reali A, Bazilevs Y, Hughes TJR (2006) Isogeometric analysis of structural vibrations. Comput Methods Appl Mech Eng 195(41):5257-5296

12. Lipton S, Evans JA, Bazilevs Y, Elguedj T, Hughes TJR (2010) Robustness of isogeometric structural discretizations under severe mesh distortion. Comput Methods Appl Mech Eng 199:357-373

13. Bardenhagen SG, Kober EM (2004) The generalized interpolation material point method. Comput Model Eng Sci 5(6):477-495

14. Bazilevs Y, Beirao da Veiga L, Cottrell JA, Hughes TJR, Sangalli $\mathrm{G}$ (2006) Isogeometric analysis: approximation, stability and error estimates for h-refined meshes. Math Models Methods Appl Sci 16(07):1031-1090

15. Cottrell JA, Hughes TJR, Bazilevs Y (2009) Isogeometric analysis: toward integration of CAD and FEA. Wiley, New York

16. Bazilevs Y, Calo VM, Zhang Y, Hughes TJR (2006) Isogeometric fluid-structure interaction analysis with applications to arterial blood flow. Comput Mech 38(4-5):310-322

17. Bazilevs Y, Calo VM, Hughes TJR, Zhang Y (2008) Isogeometric fluid-structure interaction: theory, algorithms, and computations. Comput Mech 43(1):3-37

18. Benson DJ, Bazilevs Y, Hsu M-C, Hughes TJR (2010) Isogeometric shell analysis: the Reissner-Mindlin shell. Comput Methods Appl Mech Eng 199(5):276-289

19. Cox G (1972) The numerical evaluation of b-splines. IMA J Appl Math 10(2):134-149

20. De Boor C (1972) On calculating with b-splines. J Approx Theory 6(1):50-62

21. Beirao da Veiga L, Cho D, Sangalli G (2012) Anisotropic NURBS approximation in isogeometric analysis. Comput Methods Appl Mech Eng 209-212:1-11
22. Hughes TJR, Reali A, Sangalli G (2010) Efficient quadrature for NURBS-based isogeometric analysis. Comput Methods Appl Mech Eng 199:301-313

23. Auricchio F, Calabrò F, Hughes TJR, Reali A, Sangalli G (2012) A simple algorithm for obtaining nearly optimal quadrature rules for NURBS-based isogeometric analysis. Comput Methods Appl Mech Eng 249-252:15-27

24. Nitsche J (1971) Über ein Variationsprinzip zur Lösung von Dirichlet-Problemen bei Verwendung von Teilräumen, die keinen Randbedingungen unterworfen sind. Abhandlungen aus dem Mathematischen Seminar der Universität Hamburg 36:9-15

25. Chen JS, Liu WK, Hillman M, Chi SW, Lian Y, Bessa MA (2016) Reproducing kernel approximation and discretization. In: Stein E, de Borst R, Hughes TJR (eds) Encyclopedia of computational mechanics, 2nd edn. Wiley, New York (in press)

26. Liu WK, Li S, Belytschko T (1997) Moving least-square reproducing kernel methods (I): methodology and convergence. Comput Methods Appl Mech Eng 143(1-2):113-154

27. Chen J-S, Wu C-T, Yoon S, You Y (2001) A stabilized conforming nodal integration for Galerkin mesh-free methods. Int J Numer Methods Eng 50:435-466

28. Chen JS, Hillman M, Rüter M (2013) An arbitrary order variationally consistent integration for galerkin meshfree methods. Int J Numer Methods Eng 95(5):387-418

29. Guan PC, Chi S-W, Chen JS, Slawson TR, Roth MJ (2011) Semilagrangian reproducing kernel particle method for fragment-impact problems. Int J Impact Eng 38(12):1033-1047

30. Hillman M, Chen JS, Chi SW (2014) Stabilized and variationally consistent nodal integration for meshfree modeling of impact problems. Comput Part Mech 1:245-256

31. Hillman M, Chen JS, Bazilevs Y (2015) Variationally consistent domain integration for isogeometric analysis. Comput Methods Appl Mech Eng 284:521-540

32. Hillman M, Chen JS (2016) An accelerated, convergent, and stable nodal integration in galerkin meshfree methods for linear and nonlinear mechanics. Int J Numer Methods Eng 107:603-630

33. Chen JS, Zhang X, Belytschko T (2004) An implicit gradient model by a reproducing kernel strain regularization in strain localization problems. Comput Methods Appl Mech Eng 193(27-29):28272844

34. Massing A, Larson MG, Logg A, Rognes ME (2014) A stabilized Nitsche fictitious domain method for the Stokes problem. J Sci Comput 61(3):604-628

35. Burman E (2010) Ghost penalty. Comptes Rendus Mathematique 348:1217-1220

36. Dettmer WG, Kadapa C, Peric D (2016) A stabilised immersed boundary method on hierarchical B-spline grids. Comput Methods Appl Mech Eng 311:415-437

37. Belytschko T, Liu WK, Moran B (2000) Nonlinear finite elements for continua and structures. Wiley, New York

38. Sod GA (1978) A survey of several finite difference methods for systems of nonlinear hyperbolic conservation laws. J Comput Phys 27:1-31

39. Sedov LI (1993) Similarity and dimensional methods in mechanics, 10th edn. CRC Press, Boca Raton

40. Giordano J, Jourdan G, Burtschell Y, Medale M, Zeitoun DE, Houas L (2005) Shock wave impacts on deforming panel, an application of fluid-structure interaction. Shock Waves 14(12): $103-110$

41. Deiterding R, Wood S (2013) Parallel adaptive fluid-structure interaction simulation of explosions impacting on building structures. Comput Fluids 88:719-729

42. Collier N, Dalcin L, Calo VM (2013) PetIGA: high-performance isogeometric analysis. arXiv:1305.4452 